\title{
A FUNCTIONAL FROM GEOMETRY WITH APPLICATIONS TO DISCREPANCY ESTIMATES AND THE RADON TRANSFORM
}

\author{
ALLEN D. ROGERS
}

\begin{abstract}
Estimates of discrepancy, or irregularities of distribution, are obtained for measures without atoms. Two estimators are used, the half-space, or separation, discrepancy $D_{S}$ and a geometric functional $I^{\alpha}$. A representation formula for the generalized energy integral $I^{\alpha}$ is developed. Norm inequalities for the Radon transform are obtained as an application of the continuous discrepancy results. Integral geometric notions play a prominent role.
\end{abstract}

\section{INTRODUCTION}

The study of irregularities of distribution, or discrepancy, traces its history to the fundamental paper of Weyl [We] on uniform distribution modulo one. From a general point of view, discrepancy is an indication of how a signed measure of total mass zero fails to approximate the zero measure. The subject was greatly expanded by Roth [Rt], Schmidt [Sm2], and, more recently, by Beck [BC]. The book [KN] of Kuipers and Niederreiter summarizes the history of the subject and contains an extensive bibliography. The book [BC] by Beck and Chen also presents an overview and includes many recent results.

Nearly all previous estimates in the field of irregularities of distribution have dealt with either signed atomic measures or with signed measures whose positive part is uniform and whose negative part is atomic. The present work obtains discrepancy estimates for measures with continuous positive and negative parts. Extensive use is made of a geometric functional denoted $I^{\alpha}$. Included in this work is a development of a representation formula for the generalized energy integral $I^{\alpha}$. The continuous discrepancy results are applied to obtain norm inequalities for the Radon transform.

Let $\nu$ be a compactly supported, signed Borel measure on $\mathbf{R}^{n}$ such that $\nu\left(\mathbf{R}^{n}\right)=0$. One estimator of discrepancy, the separation discrepancy $D_{S}$, is defined by

$$
D_{S}(\nu)=\sup |\nu H|
$$

where the supremum is taken over all open half-spaces $H$ of $\mathbf{R}^{n}$. A number of problems in the literature of irregularities of distribution can be expressed

Received by the editors October 16, 1991.

1991 Mathematics Subject Classification. Primary 11K38; Secondary 52A22, 44A12.

Key words and phrases. Discrepancy, irregularities of distribution, generalized energy integral, metric geometry, integral geometry, Radon transform.

This paper is based in part on the author's doctoral thesis at the University of Illinois at UrbanaChampaign. 
in terms of the separation discrepancy. For example, conjectures by Erdős [E] concerning the discrepancy of spherical caps (first solved by Schmidt [Sm1]) and the disk segment problem of Roth mentioned in [Sm2] (first solved by Beck [Be1]) have equivalent statements in terms of $D_{S}$.

The following theorem gives a lower bound for the separation discrepancy of a type of measure that does not have atoms. Note that if $N$ is even, and if $z_{i}$ is taken to be alternately \pm 1 , then one example of the measure below is a "checkerboard" of positive and negative squares.

(1.1) Theorem. Suppose the function $f$ on $\mathbf{R}^{2}$ is supported by $N^{2}$ congruent open squares $S_{i}$ arranged in an $N$ by $N$ square lattice. Let $s$ be the side length of each square, and let $f$ have value $z_{i} \in \mathbf{R}$ on $S_{i}$. Suppose further that $\sum z_{i}=0$. If $\nu$ is given by $\nu(E)=\int_{E} f$, then

$$
D_{S}(\nu) \geq c s^{2}\left(\frac{1}{N} \sum\left|z_{i}\right|^{2}\right)^{1 / 2},
$$

where $c$ is a positive constant independent of the location of the lattice and of the number, size, and weights of the squares.

As another example, the following theorem treats a measure that has a smooth density.

(1.2) Theorem. Suppose that the real-valued function $g$ on $\mathbf{R}^{2}$ is continuously differentiable with support in a disk of radius $R$ and that $\int g=0$ but $g \not \equiv 0$. If $\mu$ is given by $\mu(E)=\int_{E} g$, then

$$
D_{S}(\mu) \geq c R^{-1 / 2}\|\mid \operatorname{grad} g\|_{\infty}^{-5 / 2}\|g\|_{\infty}^{7 / 2} .
$$

where $c$ is a positive constant independent of $g$.

Theorems (1.1) and (1.2) are special cases of higher dimensional results in $\S 4.5$. We remark that Theorem (1.1) holds also for $z_{i} \in \mathbf{C}$ if complex measures $\nu$ are considered.

These results concerning the separation discrepancy follow from precise bounds on a second estimator of discrepancy, the functional $I^{\alpha}$. This functional is defined by

$$
I^{\alpha}(\nu)=\iint|x-y|^{\alpha} d \nu(x) d \nu(y),
$$

where $\nu$ is a signed measure on a compact subset of $\mathbf{R}^{n}$, and $\alpha$ is a positive real number. For example, if $\nu_{0}$ is an atomic measure assigning weight $w_{i}$ to the point $x_{i}$, then

$$
I^{\alpha}\left(\nu_{0}\right)=2 \sum_{i<j}\left|x_{i}-x_{j}\right|^{\alpha} w_{i} w_{j}
$$

If $\nu$ is required to satisfy $\nu\left(\mathbf{R}^{n}\right)=0$ and if $0<\alpha<2$, then it follows immediately from work by Schoenberg [Sol and So2] on isometric embeddings of metric spaces into Hilbert space that $-I^{\alpha}(\nu) \geq 0$. In fact, by [Bj, Lemma 1], $I^{\alpha}(\nu)=0$ if and only if $\nu$ is the zero measure. Because of this positive definiteness, the functional $-I^{\alpha}$ for $0<\alpha<2$ serves as an indicator of discrepancy. We remark that this positive definiteness is a nontrivial property; for the case $\alpha=1$, it is equivalent to the uniqueness of the Radon transform. 
Historically, the functional $I^{\alpha}$ has been of interest also in potential theory, particularly for negative $\alpha$. The classic problem is that of minimizing the energy integral $I^{2-n}(\nu)$ over positive measures $\nu$ of a given total mass on a compact set $K$ in $\mathbf{R}^{n}$ with $n>2$. Riesz and Frostman [Fr] considered generalizations to $I^{\lambda-n}(\nu)$ where $0<\lambda \leq 2$. The analogous problem of maximizing $I^{\alpha}(\nu)$ when $\alpha>0$ was investigated by Björck [Bj]. Pólya and Szegö [PS] studied the generalized energy integral $I^{\alpha}$ in their investigation of capacity and transfinite diameter.

Alexander and Stolarsky [AS] studied the problem of maximizing $I^{\alpha}(\nu)$ $(\alpha>0)$ over families of (possibly signed) measures $\nu$ of total mass one, and also considered cases where the kernel $|x-y|^{\alpha}$ is replaced by more general continuous real-valued functions on $K \times K$. Their work uses results from Schoenberg's metric embedding theory as well as techniques of Pólya and Szegö for the calculation of transfinite diameters.

In [St], Stolarsky showed that the sum of distances between $N$ points on a Euclidean unit sphere plus a quadratic mean of the spherical cap discrepancy is a constant, thereby pointing out for the first time an important relation between metric geometry and discrepancy. Stolarsky then used estimates by Schmidt [Sm1] on the discrepancy of spherical caps to estimate sums of distances.

Further insight into the functional $I^{\alpha}$ can be obtained via integral geometry. To this end, we now briefly discuss the motion-invariant measure $\eta$ on sets of lines in the plane.

It is convenient to think of the lines in $\mathbf{R}^{2}$ as oriented, by identifying an oriented line with an open half-plane. For a given oriented line in the plane, the outwardly pointing unit normal vector $\sigma$ and the algebraic distance $t$ from the line to the origin define a point $(\sigma, t)$ on the cylinder $S^{1} \times \mathbf{R}$. This correspondence is one-to-one, and surface measure on the cylinder induces the unique (up to constant multiple) motion-invariant measure $\eta$ on sets of oriented lines in the plane. With proper normalization, the $\eta$-measure of the collection of lines that intersect a bounded convex set $K$ is equal to the length of the boundary of $K$. For the degenerate case when $K$ is the line segment $\overline{x y}$, we have

$$
\eta\{h: h \cap \overline{x y} \neq \varnothing\}=2|x-y| .
$$

More generally, if $C$ is any rectifiable curve, then the integral $\int N(l, C) d \eta(l)$ is equal to twice the length of $C$, where $N(l, C)$ is the number of intersection points of $l$ with $C$. A discussion of these integral geometric notions can be found in [Sa, Chapter 3].

For the specific example when $\nu_{0}$ is area measure restricted to a bounded convex set $K$ in the plane, it can be shown [Sa, Formula 4.5, p. 47] that

$$
I^{j}\left(\nu_{0}\right)=\frac{2}{(j+2)(j+3)} \int_{l \cap K \neq \varnothing} L(l, K)^{j+3} d \eta(l), \quad j=-1,0,1,2, \ldots,
$$

where $L(l, K)$ is the length of the chord $l \cap K$. The integrals $\int L(l, K)^{j+3} d \eta(l)$ have been studied by Blaschke, Crofton, and others. Santaló [Sa, Chapter 4, $\S 2]$ gives a historical discussion with references, and also presents examples of direct computations for $j=1$. For example, if $K$ is a circle of radius $R$, then by computing $\int L(l, K)^{4} d \eta(l)$, one finds that $I^{1}\left(\nu_{0}\right)=(128 / 45) \pi R^{5}$. 
The measure $\eta$ can be generalized in an analogous manner to the motioninvariant measure on sets of oriented hyperplanes in $\mathbf{R}^{n}$ : there is a one-to-one correspondence between the collection of oriented hyperplanes in $\mathbf{R}^{n}$ and $S^{n-1}$ $\times \mathbf{R}$, and $\eta$ is induced from the product of Euclidean surface measure on $S^{n-1}$ with Lebesgue measure on $\mathbf{R}$. However, we normalize so that formula (1.3) is still valid in higher dimensions. This differs from the more usual normalization found in [Sa] but is more convenient for our purposes.

Alexander [A1] showed that the formula (1.3) leads to a representation for $I^{1}$ as an integral with respect to the measure $\eta$. He demonstrated that if $\nu$ is a compactly supported signed measure on $\mathbf{R}^{n}$, then

$$
I^{1}(\nu)=\int \nu\left(H^{+}\right) \nu\left(H^{-}\right) d \eta(h)
$$

where $H^{+}$and $H^{-}$are the open half-spaces determined by the oriented hyperplane $h$. In particular, if $\nu$ has total mass zero, then

$$
-I^{1}(\nu)=\int\left[\nu\left(H^{+}\right)\right]^{2} d \eta(h) .
$$

This formula led to recent advances in irregularities of distribution. Since $|\nu(H)| \leq D_{S}(\nu)$ for all $H$, formula (1.3) leads to the inequality

$$
-I^{1}(\nu)<c_{n} R\left[D_{S}(\nu)\right]^{2}
$$

if $\nu$ has support in a disk of radius $R$. Here $c_{n}$ is a positive dimensional constant. Recently, Alexander [A2] developed methods for finding precise bounds on $-I^{1}$ for certain classes of measures, thus giving new techniques for estimating $D_{S}$; he used these estimates to obtain an improved bound for the Roth disk segment problem.

In [A3], Alexander extended the ideas of [A2] to obtain exact bounds on $-I^{\alpha}$ $(0<\alpha<2)$ for particular classes of measures, including measures defined on arbitrary convex $(n-1)$-dimensional hypersurfaces embedded in $\mathbf{R}^{n}$. We remark that the work of Beck [Be2] contains a result that implies the bound on $-I^{1}$ for a special spherical case of [A3]. Beck used an independent method of Fourier transforms. The results of G. Wagner [Wa] include a bound on $-I^{\alpha}$ $(0<\alpha<2)$, obtained by an independent technique of spherical harmonics, that is equivalent to the special spherical case of the estimates in [A3]. In this spherical setting, Wagner also established estimates for the cases $1-n<\alpha \leq 0$, using appropriate definitions for $I^{\alpha}$ when $\alpha$ is nonpositive.

In this paper we begin in a more general setting. We shall consider measures that are complex-valued, and we shall regard $I^{\alpha}$ as a Hermitian functional by taking the outside integration with respect to $d \bar{\nu}$, where the bar denotes complex conjugation. In fact, it is natural to consider the associated functional $J^{\alpha}$, linear in the first variable and conjugate linear in the second, defined by

$$
J^{\alpha}(\mu, \nu)=\iint|x-y|^{\alpha} d \mu(x) d \bar{\nu}(y)
$$

where $\mu$ and $\nu$ are complex-valued Borel measures on $\mathbf{R}^{n}$. The following theorem is proved in $\S 3.5$. 
(1.8) Theorem. Suppose $\nu_{1}$ and $\nu_{2}$ are complex-valued Borel measures on $\mathbf{R}^{n}$ with compact support. Suppose further that $\int x^{\beta} d \nu_{j}(x)=0$ for all multiindices $\beta$ with $|\beta| \leq k$.

(a) If $0<\alpha<2 k+2$ and if $\alpha$ is not even, then

$$
J^{\alpha}\left(\nu_{1}, \nu_{2}\right)=c_{n, \alpha} \int_{\mathbf{R}^{n}} \frac{\hat{\nu}_{1}(x) \overline{\hat{\nu}}_{2}(x)}{|x|^{\alpha+n}} d x,
$$

where $c_{n, \alpha}=2^{-\alpha} \pi^{-n / 2} \Gamma\left(\frac{1}{2}(n+\alpha)\right) / \Gamma\left(-\frac{1}{2} \alpha\right)$. Here $\hat{\nu}$ is the Fourier transform of $\nu$, given by $\hat{\nu}(x)=\int e^{-i\langle x, \xi\rangle} d \nu(\xi)$.

(b) If $0<\alpha<2 k+2$ and if $\alpha$ is even, then $J^{\alpha}\left(\nu_{1}, \nu_{2}\right)=0$.

The proof uses the theory of Schwartz distributions. This representation was inspired by the treatment of the distribution $r^{\alpha}$ by Helgason [Hl]. The convergence of the integral in (1.9) is guaranteed by the hypothesis that the moments $\int x^{\beta} d \nu_{j}(x)$ vanish. For $\alpha>2$, the vanishing of these moments is an appropriate generalization of the requirement that the measure have total mass zero.

Note that if $\nu=\nu_{1}=\nu_{2}$, then formula (1.9) becomes

$$
I^{\alpha}(\nu)=c_{n, \alpha} \int \frac{|\hat{\nu}(x)|^{2}}{|x|^{\alpha+n}} d x
$$

This gives a new demonstration that $-I^{\alpha}$ (or $I^{\alpha}$, depending on the sign of $c_{n, \alpha}$ ) is positive definite on an appropriate class of $\nu$, for positive noneven $\alpha$. Also, formula (1.9) seems more helpful for some applications than the defining formula (1.7) since only a single integration is apparently involved. For example, the defining formula (1.7) does not lend itself to the problem of finding an orthonormal basis with respect to the inner product $-J^{\alpha}$ (or $J^{\alpha}$ ). However with formula (1.9), roughly speaking, an orthonormal basis $g_{j}$ with respect to the usual inner product $\int g_{i} \bar{g}_{j}$ gives rise to an orthonormal basis with respect to $\pm J^{\alpha}$ by solving $\hat{\nu}_{j}(x)=g_{j}(x)|x|^{(\alpha+n) / 2}$ for $\nu_{j}$. Details are in $\S 3.6$.

The separation discrepancy estimates for nonatomic measures follow from estimates on the functional $I^{\alpha}$, using formula (1.6). Theorems (1.1) and (1.2) are consequences of the following precise estimates.

(1.10) Theorem. Let $\nu$ be as in Theorem (1.1), with $z_{i} \in \mathbf{C}$. If $0<\alpha<2$, then

$$
-I^{\alpha}(\nu) \geq c_{\alpha} s^{4+\alpha}\left(\sum\left|z_{i}\right|^{2}\right),
$$

where $c_{\alpha}$ is a positive constant independent of the location of the lattice and of the number, size, and weights of the squares. This inequality is best possible up to choice of the constant $c_{\alpha}$.

(1.11) Theorem. Let $\mu$ be as in Theorem (1.2). If $0<\alpha<2$, then

$$
-I^{\alpha}(\mu) \geq c_{\alpha}\||\operatorname{grad} g|\|\left\|_{\infty}^{-4-\alpha}\right\| g \|_{\infty}^{6+\alpha},
$$

where $c_{\alpha}$ is a positive constant independent of the density $g$. This inequality is best possible up to the choice of the constant $c_{\alpha}$.

Theorems (1.10) and (1.11), together with their extensions to higher dimensions, are proved in $\S \S 4.2$ and 4.3 . 
Finally, there is a close relation between the Radon transform and the functional $I^{1}$. The relation can be exploited to obtain norm inequalities using the above results. Let $S^{n-1}$ be the unit sphere in $\mathbf{R}^{n}$. For a Borel measurable function $f$ on $\mathbf{R}^{n}$, the Radon transform $\mathscr{R} f$ of $f$ is defined by

$$
\mathscr{R} f(\sigma, t)=\int_{\langle x, \sigma\rangle=t} f(x) d x, \quad \sigma \in S^{n-1}, t \in \mathbf{R} .
$$

The integral here is with respect to $(n-1)$-dimensional Lebesgue measure on the hyperplane $\langle x, \sigma\rangle=t$. A mixed norm for functions $F$ on $S^{n-1} \times \mathbf{R}$ can be defined by

$$
\|F\|_{q, p}=\left(\int_{S^{n-1}}\left[\int_{-\infty}^{\infty}|F(\sigma, t)|^{p} d t\right]^{q / p} d \sigma\right)^{1 / q} .
$$

Here $d \sigma$ means integration with respect to Euclidean surface measure on $S^{n-1}$. Estimates of the type $\|\mathscr{R} f\|_{q, p} \leq C_{r, q, p}\|f\|_{r}$ have been studied by Oberlin and Stein [OS] and others. Notice that when $q=p$, this mixed norm is simply the usual $L^{p}\left(S^{n-1} \times \mathbf{R}\right)$ norm, and in particular, $\|F\|_{2,2}$ is equal to the usual $L^{2}$ norm $\|F\|_{2}$ on $S^{n-1} \times \mathbf{R}$.

Theorems (1.10) and (1.11) lead to inequalities which bound $\|\mathscr{R} f\|_{2, p}$ away from zero, as is discussed in $§ 4.6$. The following theorems illustrate the $p=2$ case.

(1.12) Theorem. Let $f$ be the generalized checkerboard function of Theorem (1.1), with $z_{i} \in \mathbf{C}$. Then

$$
\|\mathscr{R} f\|_{2} \geq c N^{-1} s^{3 / 2}\left(\sum\left|z_{i}\right|^{2}\right)^{1 / 2}
$$

(1.13) Theorem. Let $g$ be the continuously differentiable function described in Theorem (1.2). Then

$$
\|\mathscr{R} g\|_{2} \geq c R^{-1}\|\mid \operatorname{grad} g\|_{\infty}^{-5 / 2}\|g\|_{\infty}^{7 / 2} \text {. }
$$

Theorems (1.12) and (1.13) can be regarded as strengthened versions of the uniqueness theorem for the Radon transform.

Hertle $[\mathrm{Hr}]$ has obtained interesting inequalities which bound certain Sobolev norms of $\mathscr{R} f$ away from zero by other Sobolev norms of $f$. The inequality (1.13) obtained here apparently neither implies nor follows from his.

The material in this paper is based in part on the author's doctoral thesis [Rg]. The author would like to express his gratitude to his advisor, Professor Ralph Alexander.

\section{Preliminaries}

2.1. Summary of results from measure theory. We recall some definitions and results from the theory of complex measures. For further details, we refer the reader to [DS, Fo, and $\mathrm{Ru}]$.

Throughout this paper, unless stated otherwise, it is understood that all measures are Borel measures, all sets are Borel sets, all measurable functions are complex-valued and Borel measurable, and all work is done on $\mathbf{R}^{n}$. A complex measure takes values in $\mathbf{C}$; infinite values are not allowed, so a positive or signed measure is a complex measure if and only if it is finite. Let $|\nu|$ denote 
the total variation of $\nu$, and let $M\left(\mathbf{R}^{n}\right)$ denote the Banach space of all complex measures, with norm $\|\nu\|=|\nu|\left(\mathbf{R}^{n}\right)$. We have $L^{1}(\nu)=L^{1}(|\nu|)$. A set $E$ is called $\nu$-null if $|\nu|(E)=0$. Let $\nu_{R}$ and $\nu_{I}$ denote the real and imaginary parts of $\nu$, respectively, and define $\bar{\nu}=\nu_{R}-i \nu_{I}$.

We shall often make use of the estimate

$$
\left|\int f d \nu\right| \leq \int|f| d|\nu|, \quad f \in L^{1}(\nu)
$$

We use $m$ to denote $n$-dimensional Lebesgue measure on the Borel sets of $\mathbf{R}^{n}$, we use $d x$ to denote integration with respect to the measure $m$, and we use $L^{1}\left(\mathbf{R}^{n}\right)$ interchangeably with $L^{1}(m)$. The notation $d \nu=f d x \quad(f \in$ $\left.L^{1}\left(\mathbf{R}^{n}\right)\right)$ indicates that $\nu(E)=\int_{E} f(x) d x$ for all $E$. If $d \nu=f d x$, then $d|\nu|=|f| d x$. By the Radon-Nikodym Theorem, the mapping $f \rightarrow f d x$ is an isometric embedding of the space $L^{1}\left(\mathbf{R}^{n}\right)$ into $M\left(\mathbf{R}^{n}\right)$, with range consisting of precisely the absolutely continuous complex measures.

If $\mu \in M\left(\mathbf{R}^{m}\right)$ and $\nu \in M\left(\mathbf{R}^{n}\right)$, then the product measure $\mu \times \nu$ is the unique complex measure on $\mathbf{R}^{m+n}$ such that

$$
(\mu \times \nu)(E \times F)=\mu(E) \nu(F)
$$

for all $E \subset \mathbf{R}^{m}, F \subset \mathbf{R}^{n}$. We have $|\mu \times \nu|=|\mu| \times|\nu|$. In particular, $L^{1}(\mu \times \nu)=$ $L^{1}(|\mu| \times|\nu|)$.

The convolution of two complex measures $\mu$ and $\nu$ is the complex measure $\mu * \nu$ defined by

$$
(\mu * \nu)(E)=\iint \chi_{E}(x+y) d \mu(x) d \nu(y) .
$$

If $f \in L^{1}\left(\mathbf{R}^{n}\right)$ and $\nu \in M\left(\mathbf{R}^{n}\right)$, then $f * \nu \in L^{1}\left(\mathbf{R}^{n}\right)$ is given by

$$
(f * \nu)(x)=\int f(x-y) d \nu(y) .
$$

If $f, g \in L^{1}\left(\mathbf{R}^{n}\right)$, then $f * g \in L^{1}\left(\mathbf{R}^{n}\right)$ is given by

$$
(f * g)(x)=\int f(x-y) g(y) d y .
$$

The latter two definitions agree with the first when $L^{1}\left(\mathbf{R}^{n}\right)$ is identified with a subspace of $M\left(\mathbf{R}^{n}\right)$ under the map $f \rightarrow f d x$. The last equation defines $f * g$ for functions $f$ and $g$ that are not necessarily in $L^{1}\left(\mathbf{R}^{n}\right)$, provided the integral exists.

A multi-index is an ordered $n$-tuple of nonnegative integers. If $\beta=\left(\beta_{1}, \ldots\right.$, $\left.\beta_{n}\right)$ is a multi-index and $x=\left(x_{1}, \ldots, x_{n}\right) \in \mathbf{R}^{n}$, then define

$$
\begin{aligned}
|\beta| & =\beta_{1}+\cdots+\beta_{n}, \\
\beta ! & =\beta_{1} ! \cdots \beta_{n} !, \\
x^{\beta} & =x_{1}^{\beta_{1}} \cdots x_{n}^{\beta_{n}}, \\
\partial^{\beta} & =\partial_{x}^{\beta}=\left(\frac{\partial}{\partial x_{1}}\right)^{\beta_{1}} \cdots\left(\frac{\partial}{\partial x_{n}}\right)^{\beta_{n}} .
\end{aligned}
$$

2.2. The classes $M^{t}\left(\mathbf{R}^{n}\right)$ and $V^{k}\left(\mathbf{R}^{n}\right)$. In this section we discuss certain classes of complex measures and their behavior under convolution. For real 
$t \geq 0$, define

$$
M^{t}\left(\mathbf{R}^{n}\right)=\left\{\nu \in M\left(\mathbf{R}^{n}\right): \int|x|^{t} d|\nu|(x)<\infty\right\} .
$$

That is, $\nu \in M^{t}\left(\mathbf{R}^{n}\right)$ if and only if $|x|^{t} \in L^{1}(\nu)$. For $0 \leq s \leq t$, we have $M^{s}\left(\mathbf{R}^{n}\right) \supset M^{t}\left(\mathbf{R}^{n}\right)$. Define

$$
M^{\infty}\left(\mathbf{R}^{n}\right)=\bigcap_{k=1}^{\infty} M^{k}\left(\mathbf{R}^{n}\right)
$$

and

$$
M_{c}\left(\mathbf{R}^{n}\right)=\left\{\nu \in M\left(\mathbf{R}^{n}\right): \nu \text { has compact support }\right\} .
$$

Example. Let $f$ be in the space $\mathscr{S}\left(\mathbf{R}^{n}\right)$ of infinitely differentiable functions which, together with all of their derivatives, remain bounded when multiplied by any polynomial. Then the measure $\nu$ given by $d \nu=f d x$ is in $M^{\infty}\left(\mathbf{R}^{n}\right)$.

We remark that, of these spaces, only $M\left(\mathbf{R}^{n}\right)$ is complete (with respect to the norm $\left.\|\nu\|=|\nu|\left(\mathbf{R}^{n}\right)\right)$. Indeed, $C_{c}^{\infty}\left(\mathbf{R}^{n}\right)$ is dense in the (isometrically embedded) subspace $L^{1}\left(\mathbf{R}^{n}\right)$ of $M\left(\mathbf{R}^{n}\right)$. Thus, for fixed $t>0$, there exist functions in $C_{c}^{\infty}\left(\mathbf{R}^{n}\right)$ (hence, measures in $M_{c}\left(\mathbf{R}^{n}\right)$ ) which converge to a measure in $L^{1}\left(\mathbf{R}^{n}\right) \subset M\left(\mathbf{R}^{n}\right)$ but not in $M^{t}\left(\mathbf{R}^{n}\right)$.

(2.2.1) Lemma. Let $k$ be a nonnegative integer. Then there exist positive constants $c_{n, k}$ and $C_{n, k}$ such that

$$
c_{n, k}|x|^{k} \leq \sum_{|\beta|=k}\left|x^{\beta}\right| \leq C_{n, k}|x|^{k}, \text { for all } x \in \mathbf{R}^{n} .
$$

Proof. On the compact set $\left\{y \in \mathbf{R}^{n}:|y|=1\right\}$, the continuous function $\sum_{|\beta|=k}\left|y^{\beta}\right|$ is always positive. Therefore, let $c_{n, k}>0$ be the minimum of $\sum_{|\beta|=k}\left|y^{\beta}\right|$ on $\{y:|y|=1\}$ and let $C_{n, k}$ be the maximum. The lemma follows by homogeneity.

The lemma shows the equivalence of the condition $|x|^{k} \in L^{1}(|\nu|)$ with the condition that $x^{\beta} \in L^{1}(|\nu|)$ for all multi-indices $\beta$ such that $|\beta|=k$.

Spaces of measures with vanishing moments will also be of interest. For each nonnegative integer $k$, define

$$
V^{k}\left(\mathbf{R}^{n}\right)=\left\{\nu \in M^{k}\left(\mathbf{R}^{n}\right): \int x^{\beta} d \nu(x)=0,0 \leq|\beta| \leq k\right\}
$$

and

$$
V^{\infty}\left(\mathbf{R}^{n}\right)=\bigcap_{k=1}^{\infty} V^{k}\left(\mathbf{R}^{n}\right) .
$$

We now establish that $M^{t}\left(\mathbf{R}^{n}\right)$ is closed under convolution, and that $V^{k}\left(\mathbf{R}^{n}\right)$ is an ideal in $M^{k}\left(\mathbf{R}^{n}\right)$. The following lemma is a standard result in the case when $f$ is bounded (in which case the hypothesis on $g$ is satisfied a priori). 
(2.2.2) Lemma. Let $f$ be a complex-valued measurable function on $\mathbf{R}^{n}$, and let $\mu, \nu \in M\left(\mathbf{R}^{n}\right)$. Suppose the function $g$ on $\mathbf{R}^{2 n}$ given by $g(x, y)=f(x+y)$ is in $L^{1}(\mu \times \nu)$. Then $f \in L^{1}(\mu * \nu)$ and

$$
\int f d(\mu * \nu)=\iint f(x+y) d \mu(x) d \nu(y) \text {. }
$$

Proof. From the definition of convolution, it follows that $|\mu| *|\nu|$ is a positive measure satisfying $|(\mu * \nu)(E)| \leq(|\mu| *|\nu|)(E)$ for all $E$. But $|\mu * \nu|$ is the least such positive measure, so $|\mu * \nu|(E) \leq(|\mu| *|\nu|)(E)$ for all $E$. Therefore,

$$
\int h d|\mu * \nu| \leq \int h d(|\mu| *|\nu|)=\iint h(x+y) d|\mu|(x) d|\nu|(y)
$$

when $h$ is a characteristic function, and hence, by linearity and the Monotone Convergence Theorem, when $h$ is a nonnegative measurable function on $\mathbf{R}^{n}$. In particular, taking $h=|f|$ shows that $f \in L^{1}(\mu * \nu)$.

Equation (2.2.3) holds when $f$ is a characteristic function. The general case follows by linearity and the Dominated Convergence Theorem.

(2.2.4) Proposition. The class $M^{t}\left(\mathbf{R}^{n}\right)$ is closed under convolution.

Proof. Letting $f(x)=|x|^{t}$ and $g(x, y)=f(x+y)$, then by the previous lemma, it is enough to show $g \in L^{1}(\mu \times \nu)=L^{1}(|\mu| \times|\nu|)$, where $\mu$ and $\nu$ are in $M^{t}\left(\mathbf{R}^{n}\right)$. But this follows from the fact that

$$
|x+y|^{t} \leq(|x|+|y|)^{t} \leq[2 \max (|x|,|y|)]^{t} \leq 2^{t}\left(|x|^{t}+|y|^{t}\right) .
$$

(2.2.5) Proposition. The class $V^{k}\left(\mathbf{R}^{n}\right)$ is an ideal under convolution in $M^{k}\left(\mathbf{R}^{n}\right)$.

Proof. Suppose $\mu \in V^{k}\left(\mathbf{R}^{n}\right)$ and $\nu \in M^{k}\left(\mathbf{R}^{n}\right)$. Let $\beta$ be a multi-index with $|\beta| \leq k$. As in the proof of the previous proposition, $g(x, y)=|x+y|^{k} \in$ $L^{1}(\mu \times \nu)$, so that by Lemma (2.2.1), $(x+y)^{\beta} \in L^{1}(\mu \times \nu)$. Therefore by Lemma (2.2.2), $x^{\beta} \in L^{1}(\mu * \nu)$ and

$$
\int x^{\beta} d(\mu * \nu)(x)=\iint(x+y)^{\beta} d \mu(x) d \nu(y) .
$$

From the $n$-dimensional binomial theorem, it follows that

$$
\int x^{\beta} d(\mu * \nu)(x)=\sum_{\gamma+\delta=\beta} \frac{\beta !}{\gamma ! \delta !} \int x^{\gamma} d \mu(x) \int y^{\delta} d \nu(y) .
$$

Since $\int x^{\gamma} d \mu(x)=0$ whenever $|\gamma| \leq|\beta| \leq k$, we conclude $\mu * \nu \in V^{k}\left(\mathbf{R}^{n}\right)$.

2.3. The Fourier transform. The Fourier transform of a complex measure $\nu$ is the bounded continuous function $\hat{\nu}$ given by

$$
\hat{\nu}(y)=\int_{\mathbf{R}^{n}} e^{-i\langle x, y\rangle} d \nu(x), \quad y \in \mathbf{R}^{n},
$$

where $\langle x, y\rangle$ is the usual Euclidean inner product on $\mathbf{R}^{n}$. In particular, when $f \in L^{1}\left(\mathbf{R}^{n}\right)$,

$$
\hat{f}(y)=\int_{\mathbf{R}^{n}} e^{-i\langle x, y\rangle} f(x) d x, \quad y \in \mathbf{R}^{n} .
$$


With this normalization, the inverse transform is

$$
\check{g}(x)=(2 \pi)^{-n} \int_{\mathbf{R}^{n}} e^{i\langle x, y\rangle} g(y) d y, \quad x \in \mathbf{R}^{n}, g \in L^{1}\left(\mathbf{R}^{n}\right) .
$$

By applying Lemma (2.2.2) to the bounded function $f(x)=e^{-i\langle x, \xi\rangle}$, we obtain

$$
\widehat{\mu * \nu}(\xi)=\hat{\mu}(\xi) \hat{\nu}(\xi), \quad \xi \in \mathbf{R}^{n} \text {. }
$$

(2.3.1) Lemma. Let $k$ be a nonnegative integer. If $\nu \in M^{k}\left(\mathbf{R}^{n}\right)$, then $\hat{\nu} \in C^{k}$ and $\partial^{\beta} \hat{\nu}=\left((-i x)^{\beta} \nu\right)^{\wedge}$.

Proof. Note that $\left|e^{-i\langle x, y\rangle}(-i x)^{\beta}\right| \leq\left|x^{\beta}\right|$, and $x^{\beta} \in L^{1}(\nu)$ for $|\beta| \leq k$. Therefore by repeated differentiation under the integral sign (cf. [Fo, Theorem 2.27]), we have

$$
\partial_{y}^{\beta} \hat{\nu}(y)=\partial_{y}^{\beta} \int e^{-i\langle x, y\rangle} d \nu(x)=\int e^{-i\langle x, y\rangle}(-i x)^{\beta} d \nu(x) .
$$

2.4. Tempered distributions. This section contains a brief summary of some definitions and notation from the theory of tempered distributions that are used in $\S \S 3.3$ and 3.6. For further details, see [Fo, Chapter 8, $\S 5]$ and [Hl, Chapter I, $\S 2.8]$.

Let $\mathscr{S}\left(\mathbf{R}^{n}\right)$ denote the space of infinitely differentiable complex-valued functions on $\mathbf{R}^{n}$, all of whose derivatives remain bounded when multiplied by any polynomial. Equivalently, for any nonnegative integer $N$ and any multiindex $\beta$, define

$$
\|\psi\|_{(N, \beta)}=\sup _{x \in \mathbf{R}^{n}}(1+|x|)^{N}\left|\partial^{\beta} \psi(x)\right|
$$

and

$$
\mathscr{S}\left(\mathbf{R}^{n}\right)=\left\{\psi \in C^{\infty}\left(\mathbf{R}^{n}\right):\|\psi\|_{(N, \beta)}<\infty \text { for all } N, \beta\right\} .
$$

A sequence $\psi_{k}$ of functions in $\mathscr{S}\left(\mathbf{R}^{n}\right)$ converges to $\psi \in \mathscr{S}\left(\mathbf{R}^{n}\right)$ if for all $N$ and $\beta$,

$$
\left\|\psi_{k}-\psi\right\|_{(N, \beta)} \rightarrow 0 \quad \text { as } k \rightarrow \infty \text {. }
$$

A tempered distribution is a continuous linear functional on $\mathscr{S}\left(\mathbf{R}^{n}\right)$, and the set of all tempered distributions is denoted by $\mathscr{S}^{\prime}\left(\mathbf{R}^{n}\right)$. For $T \in \mathscr{S}^{\prime}\left(\mathbf{R}^{n}\right)$ and $\psi \in \mathscr{S}\left(\mathbf{R}^{n}\right)$, denote the value of $T$ at $\psi$ by $\langle T, \psi\rangle$.

A measurable function $f$ on $\mathbf{R}^{n}$ is called a tempered function if $(1+|x|)^{-N} f \in L^{1}\left(\mathbf{R}^{n}\right)$ for some $N \geq 0$, and any tempered function $f$ defines a tempered distribution $T_{f}$ by

$$
\left\langle T_{f}, \psi\right\rangle=\int f \psi, \quad \psi \in \mathscr{S}\left(\mathbf{R}^{n}\right) .
$$

It is common to identify $f$ with $T_{f}$. Any $f \in L^{p}\left(\mathbf{R}^{n}\right) \quad(1 \leq p \leq \infty)$ is a tempered function. Similarly, any complex measure $\nu$ on $\mathbf{R}^{n}$ defines a tempered distribution $T_{\nu}$ by

$$
\left\langle T_{\nu}, \psi\right\rangle=\int \psi d \nu, \quad \psi \in \mathscr{S}\left(\mathbf{R}^{n}\right)
$$

The Fourier transform of a complex measure or of a function in $L^{1}\left(\mathbf{R}^{n}\right)$ is defined in $\S 2.3$. For $\psi \in \mathscr{S}\left(\mathbf{R}^{n}\right) \subset L^{1}\left(\mathbf{R}^{n}\right)$, the transform $\hat{\psi}$ is also in $\mathscr{S}\left(\mathbf{R}^{n}\right)$. 
For $T \in \mathscr{S}^{\prime}\left(\mathbf{R}^{n}\right)$, its Fourier transform is the tempered distribution $\hat{T}$ defined by

$$
\langle\hat{T}, \psi\rangle=\langle T, \hat{\psi}\rangle, \quad \psi \in \mathscr{S}\left(\mathbf{R}^{n}\right) .
$$

As a mapping from $\mathscr{S}^{\prime}\left(\mathbf{R}^{n}\right)$ to $\mathscr{S}^{\prime}\left(\mathbf{R}^{n}\right)$, the Fourier transform is one-to-one. For $f \in L^{1}\left(\mathbf{R}^{n}\right)$, the tempered distributions $\hat{T}_{f}$ and $T_{\hat{f}}$ concide. Similarly, if $\nu$ is a complex measure, then $\hat{\nu}$ is a bounded continuous (hence, tempered) function, and $\hat{T}_{\nu}=T_{\hat{\nu}}$.

For $f \in \mathscr{S}\left(\mathbf{R}^{n}\right)$ and $T \in \mathscr{S}^{\prime}\left(\mathbf{R}^{n}\right)$, define $f T$ by

$$
\langle f T, \psi\rangle=\langle T, f \psi\rangle, \quad \psi \in \mathscr{S}\left(\mathbf{R}^{n}\right) .
$$

One can also define the tempered function (hence, tempered distribution) $f * T$; for the special case $T=T_{g}$ (where $g$ is a tempered function), $f * T_{g}$ is the tempered function $f * g$ (or the tempered distribution $T_{f * g}$ ). This convolution has the property

$$
\widehat{f * T}=\hat{f} \hat{T} .
$$

We now discuss the tempered distribution $r^{\alpha}$, as presented in [Hl]. Let $\Omega$ be an open subset of $\mathbf{C}$. A mapping $\alpha \rightarrow T_{\alpha}$ from $\Omega$ into $\mathscr{S}^{\prime}\left(\mathbf{R}^{n}\right)$ is called a meromorphic distribution-valued function if for every $\psi \in \mathscr{S}\left(\mathbf{R}^{n}\right)$, the mapping $\alpha \rightarrow\left\langle T_{\alpha}, \psi\right\rangle$ from $\Omega$ into $\mathbf{C}$ is a meromorphic function.

For $\operatorname{Re} \alpha>-n$, the function $f(x)=|x|^{\alpha}$ on $\mathbf{R}^{n}$ is a tempered function and thus defines a tempered distribution $r^{\alpha}$ by

$$
\left\langle r^{\alpha}, \psi\right\rangle=\int_{\mathbf{R}^{n}}|x|^{\alpha} \psi(x) d x, \quad \psi \in \mathscr{S}\left(\mathbf{R}^{n}\right) .
$$

The discussion surrounding [Hl, Lemma 2.33, p. 133] shows that the mapping $\alpha \rightarrow r^{\alpha}$ extends uniquely to a meromorphic mapping from $\mathbf{C}$ to $\mathscr{S}^{\prime}\left(\mathbf{R}^{n}\right)$, with simple poles at the points $\alpha=-n-2 h(h=0,1,2, \ldots)$.

If neither $\alpha$ nor $-\alpha-n$ are nonnegative even integers, then by [Hl, Lemma 2.34 , p. 134],

$$
\widehat{r}^{\alpha}=2^{n+\alpha} \pi^{n / 2} \frac{\Gamma\left(\frac{1}{2}(n+\alpha)\right)}{\Gamma\left(-\frac{1}{2} \alpha\right)} r^{-\alpha-n} .
$$

It follows from the definition of this extension that if $\psi \in \mathscr{S}\left(\mathbf{R}^{n}\right)$ and $\alpha>0$ is not an even integer, then the value of $\left\langle r^{-\alpha-n}, \psi\right\rangle$ agrees with

$$
\int_{\mathbf{R}^{n}} \frac{\psi(x)}{|x|^{\alpha+n}} d x
$$

whenever this integral is absolutely convergent.

\section{THEORY OF THE FUNCTIONAL $J^{\alpha}$}

3.1. Definition of the functional $J^{\alpha}$. Let $\alpha$ be a positive real number. For $\mu, \nu \in M^{\alpha}\left(\mathbf{R}^{n}\right)$, define the functional $J^{\alpha}$ by

$$
J^{\alpha}(\mu, \nu)=\iint|x-y|^{\alpha} d \mu(x) d \bar{\nu}(y) .
$$

The hypothesis $\mu, \nu \in M^{\alpha}\left(\mathbf{R}^{n}\right)$ guarantees that $J^{\alpha}(|\mu|,|\nu|)$ is finite, since $|x-y|^{\alpha} \leq 2^{\alpha}\left(|x|^{\alpha}+|y|^{\alpha}\right)$. The Fubini theorem may therefore be applied to interchange the integrations in (3.1.1). 
For $\lambda, \mu, \nu \in M^{\alpha}\left(\mathbf{R}^{n}\right)$ and $c \in \mathbf{C}$, we have

$$
\begin{aligned}
& J^{\alpha}(c \lambda+\mu, \nu)=c J^{\alpha}(\lambda, \nu)+J^{\alpha}(\mu, \nu), \\
& J^{\alpha}(\lambda, c \mu+\nu)=\bar{c} J^{\alpha}(\lambda, \mu)+J^{\alpha}(\lambda, \nu),
\end{aligned}
$$

and

$$
J^{\alpha}(\mu, \nu)=\overline{J^{\alpha}(\nu, \mu)}
$$

For $\nu \in M^{\alpha}\left(\mathbf{R}^{n}\right)$, define $I^{\alpha}(\nu)=J^{\alpha}(\nu, \nu)$. Observe that for $c \in \mathbf{C}, I^{\alpha}(c \nu)=$ $|c|^{2} I^{\alpha}(\nu)$. In particular, if $|c|=1$, then $I^{\alpha}(c \nu)=I^{\alpha}(\nu)$.

We now consider the motion invariance of $J^{\alpha}$ and the effects of scaling. A function $\tau: \mathbf{R}^{n} \rightarrow \mathbf{R}^{n}$ is a similarity transformation if it is of the form $\tau(x)=T x+w$, where $w \in \mathbf{R}^{n}$ and $T$ is a (necessarily invertible) linear transformation satisfying $|T x|=s|x|$ for some similarity factor $s>0$. Observe that $|\tau(x-y)|=s|x-y|$ for any $x, y \in \mathbf{R}^{n}$. If $s=1$, then $\tau$ is a motion. If $w=0$ and $\tau(x)=T x=s x$, then $\tau$ is a dilation by the factor $s$.

For a similarity transformation $\tau$, define the measure $\tau \nu$ by

$$
\tau \nu(E)=\nu\left(\tau^{-1} E\right) \text {. }
$$

If $\nu$ is a complex measure and $\tau$ is a similarity transformation, then $\overline{\tau \nu}=\tau \bar{\nu}$ and $|\tau \nu|=\tau|\nu|$. If $f \in L^{1}(\nu)$, then a linearity and approximation argument shows that $f \circ \tau^{-1} \in L^{1}(\tau \nu)$ and

$$
\int f d \nu=\int f \circ \tau^{-1} d(\tau \nu)
$$

(3.1.3) Lemma. Let $\tau$ be a similarity transformation. If $\nu \in M^{\alpha}\left(\mathbf{R}^{n}\right)$, then $\tau \nu \in M^{\alpha}\left(\mathbf{R}^{n}\right)$.

Proof. By definition, $\nu \in M^{\alpha}\left(\mathbf{R}^{n}\right)$ is equivalent to $|x|^{\alpha} \in L^{1}(\nu)$. Thus, $\left|\tau^{-1} x\right|^{\alpha} \in L^{1}(\tau \nu)$. If $\tau(x)=T x+w$ with $|T x|=s|x|$, then $x=T\left(\tau^{-1} x\right)+w$, and so $|x| \leq s\left|\tau^{-1} x\right|+|w|$. Therefore, the finiteness of $\int\left|\tau^{-1} x\right|^{\alpha} d|\tau \nu|$ implies the finiteness of $\int|x|^{\alpha} d|\tau \nu|$, and the lemma follows from (3.1.2).

The motion invariance of $J^{\alpha}$ is shown by taking $s=1$ in the following proposition.

(3.1.4) Proposition. Let $\mu, \nu \in M^{\alpha}\left(\mathbf{R}^{n}\right)$ and let $\tau$ be a similarity transformation with similarity factor $s$. Then

$$
J^{\alpha}(\tau \mu, \tau \nu)=s^{\alpha} J^{\alpha}(\mu, \nu)
$$

Proof. By (3.1.2), we have

$$
\begin{aligned}
J^{\alpha}(\tau \mu, \tau \nu) & =s^{\alpha} \iint\left|\tau^{-1} x-\tau^{-1} y\right|^{\alpha} d(\tau \mu)(x) d(\overline{\tau \nu})(y) \\
& =s^{\alpha} J^{\alpha}(\mu, \nu) .
\end{aligned}
$$

Another useful type of scaling is as follows. Define the measure $\nu_{(s)}$ by $\nu_{(s)}=s^{n} \tau \nu$, where $\tau$ is dilation by $s>0$. By linearity and Proposition (3.1.4), we have the following.

(3.1.5) Corollary. If $\mu, \nu \in M^{\alpha}\left(\mathbf{R}^{n}\right)$, then

$$
J^{\alpha}\left(\mu_{(s)}, \nu_{(s)}\right)=s^{2 n+\alpha} J^{\alpha}(\mu, \nu) .
$$

The next lemma shows how the scaled measure $\nu_{(s)}$ arises naturally. 
(3.1.6) Lemma. Let $f \in L^{1}\left(\mathbf{R}^{n}\right)$, and let $d \nu=f d x$. Define $f_{(s)}$ to be the dilation of the function $f$ by a factor of $s>0$, given by

$$
f_{(s)}(x)=f\left(s^{-1} x\right) \text {. }
$$

Then

$$
d \nu_{(s)}=f_{(s)} d x
$$

Proof. Let $\tau$ be dilation by $s>0$. Then for all $E$,

$$
\begin{aligned}
\nu_{(s)}(E) & =s^{n} \int_{\tau^{-1} E} f(x) d x=s^{n}\left|\operatorname{det}\left(\tau^{-1}\right)\right| \int_{E} f \circ \tau^{-1}(x) d x \\
& =\int_{E} f_{(s)}(x) d x .
\end{aligned}
$$

\subsection{The case of even $\alpha$.}

(3.2.1) Theorem. Suppose $\mu$ and $\nu$ are in $M^{2 k+2}\left(\mathbf{R}^{n}\right)$ and $V^{k}\left(\mathbf{R}^{n}\right)$. If $0<$ $\alpha<2 k+2$ and if $\alpha$ is even, then

$$
J^{\alpha}(\mu, \nu)=0 .
$$

Proof. Write $\alpha=2 h$, where $0<h \leq k$. Then by the multinomial theorem,

$$
|x-y|^{2 h}=\left[\left(x_{1}-y_{1}\right)^{2}+\cdots+\left(x_{n}-y_{n}\right)^{2}\right]^{h}=\sum_{|\beta|=h} \frac{h !}{\beta !}(x-y)^{2 \beta},
$$

and by the $n$-dimensional binomial theorem,

$$
(x-y)^{2 \beta}=\sum_{\gamma+\dot{\delta}=2 \beta}(-1)^{|\delta|} \frac{(2 \beta) !}{\gamma ! \delta !} x^{\gamma} y^{\delta} .
$$

Thus,

$$
\begin{aligned}
J^{2 h}(\mu, \nu) & =\iint|x-y|^{2 h} d \mu(x) d \bar{\nu}(y) \\
& =\sum_{|\beta|=h} \sum_{\gamma+\delta=2 \beta}(-1)^{|\delta|} \frac{h !(2 \beta) !}{\beta ! \gamma ! \delta !}\left(\int x^{\gamma} d \mu(x)\right)\left(\int y^{\delta} d \bar{\nu}(y)\right) .
\end{aligned}
$$

Now for each $\gamma$ and $\delta$ with $\gamma+\delta=2 \beta$ and $|\beta|=h$, we have $|\gamma|+|\delta|=2 h$, so that either $\int x^{\gamma} d \mu(x)$ or $\int y^{\delta} d \bar{\nu}(y)$ is zero. Therefore $J^{2 h}(\mu, \nu)=0$.

3.3. A representation formula. The proof of the following theorem uses results about the space $\mathscr{S}^{\prime}\left(\mathbf{R}^{n}\right)$ of tempered distributions. We remark that the vanishing of the moments $\int x^{\beta} f_{j}(x) d x$ in the following is exactly the condition necessary to guarantee the absolute convergence of the integral (3.3.2).

(3.3.1) Theorem. Suppose $f_{1}, f_{2} \in \mathscr{S}\left(\mathbf{R}^{n}\right)$ with $\int x^{\beta} f_{j}(x) d x=0$ for all multiindices $\beta$ with $|\beta| \leq k$. Let $d \nu_{j}=f_{j} d x$. If $0<\alpha<2 k+2$ and if $\alpha$ is not even, then

$$
J^{\alpha}\left(\nu_{1}, \nu_{2}\right)=c_{n, \alpha} \int_{\mathbf{R}^{n}} \frac{\hat{f}_{1}(x) \overline{\hat{f}}_{2}(x)}{|x|^{\alpha+n}} d x,
$$

where $c_{n, \alpha}=2^{-\alpha} \pi^{-n / 2} \Gamma\left(\frac{1}{2}(n+\alpha)\right) / \Gamma\left(-\frac{1}{2} \alpha\right)$. 
Proof. Consider

$$
J^{\alpha}\left(\nu_{1}, \nu_{2}\right)=\iint|x-y|^{\alpha} f_{1}(x) \overline{f_{2}(y)} d x d y .
$$

Now

$$
\int|x-y|^{\alpha} f_{1}(x) d x=f_{1} * r^{\alpha}(y)
$$

where the function $r^{\alpha}$ for $\alpha>0$ is given by $r^{\alpha}(y)=|y|^{\alpha}$. But for $\alpha>0, r^{\alpha}$ can also be regarded as a tempered distribution whose value at $\psi \in \mathscr{S}\left(\mathbf{R}^{n}\right)$ is $\left\langle r^{\alpha}, \psi\right\rangle=\int|x|^{\alpha} \psi(x) d x$. Therefore $f_{1} * r^{\alpha}$ is a tempered distribution, and we have by the theory of distributions

$$
\begin{aligned}
J^{\alpha}\left(\nu_{1}, \nu_{2}\right) & =\left\langle f_{1} * r^{\alpha}, \bar{f}_{2}\right\rangle=\left\langle\widehat{f_{1} * r^{\alpha}}, \check{\bar{f}}_{2}\right\rangle=\left\langle\hat{f}_{1} \widehat{r}^{\alpha}, \check{\bar{f}}_{2}\right\rangle \\
& =\left\langle\widehat{r}^{\alpha}, \hat{f}_{1} \check{\bar{f}}_{2}\right\rangle=(2 \pi)^{-n}\left\langle\widehat{r}^{\alpha}, \hat{f}_{1} \overline{\hat{f}}_{2}\right\rangle .
\end{aligned}
$$

Now since $\alpha$ is not even, we have by [H1, Lemma 2.34, p. 134] that

$$
\widehat{r^{\alpha}}=C_{n, \alpha} r^{-\alpha-n}
$$

where

$$
C_{n, \alpha}=2^{n+\alpha} \pi^{n / 2} \frac{\Gamma\left(\frac{1}{2}(n+\alpha)\right)}{\Gamma\left(-\frac{1}{2} \alpha\right)} .
$$

The distribution $r^{-\alpha-n}$ is discussed in $\S 2.4$. The value of $\left\langle r^{-\alpha-n}, \hat{f}_{1} \overline{\hat{f}}_{2}\right\rangle$ agrees with

$$
\int_{\mathbf{R}^{n}} \frac{\hat{f}_{1}(x) \overline{\hat{f}}_{2}(x)}{|x|^{\alpha+n}} d x
$$

whenever this integral is absolutely convergent. To establish absolute convergence, it suffices in this case to consider the behavior of the integrand near the origin. Note that the vanishing of $\int x^{\beta} f_{j}(x) d x$ is equivalent to the vanishing of $\partial^{\beta} \hat{f}_{j}(0)$ by Lemma (2.3.1). Therefore by Taylor's formula, we have

$$
\hat{f}_{j}(x)=\sum_{|\beta|=k+1} a_{j, \beta} x^{\beta}+R_{j}(x),
$$

for some $a_{j, \beta}$, where $\left|R_{j}(x)\right| \leq c_{j}|x|^{k+1}$ on $\{x:|x| \leq 1\}$ for some $c_{j}$. From Lemma (2.2.1), it follows that $\left|\hat{f}_{j}(x)\right| \leq C_{j}|x|^{k+1}$ on $\{x:|x| \leq 1\}$ for some $C_{j}$; hence,

$$
\frac{\left|\hat{f}_{1}(x) \overline{\hat{f}}_{2}(x)\right|}{|x|^{\alpha+n}}<\frac{C|x|^{2 k+2}}{|x|^{\alpha+n}}, \quad|x| \leq 1,
$$

for some $C$. Since $\alpha<2 k+2$, the integral (3.3.3) converges absolutely, and the theorem is proved.

3.4. Weak convergence results. In this section, we discuss topics closely related to the weak convergence of measures. These results will be used to generalize Theorem (3.3.1). Items (3.4.1)-(3.4.3) are taken from Bochner [Bo]. 
(3.4.1) Definition. A sequence $\left\{\nu_{l}\right\}$ of complex measures is said to be Bernoulli convergent to the complex measure $\nu$ if

$$
\lim _{l \rightarrow \infty} \int f d \nu_{l}=\int f d \nu
$$

for all bounded continuous $f: \mathbf{R}^{n} \rightarrow \mathbf{C}$.

(The requirement in [Bo] that the norms $\left\|\nu_{l}\right\|$ be jointly bounded is superfluous, by the Uniform Boundedness Principle [Fo, Theorem 5.12].)

Throughout this section, if $\psi$ is any function on $\mathbf{R}^{n}$, then define

$$
\psi_{l}(x)=l^{n} \psi(l x)
$$

for all positive integers $l$. The following lemma is from [Bo, $\S 1.1]$.

(3.4.2) Lemma. Let $\psi \in L^{1}\left(\mathbf{R}^{n}\right)$ with $\int \psi(x) d x=1$. Then

(a) $\int\left|\psi_{l}(x)\right| d x=\int|\psi(x)| d x$ for all $l$, and

(b) $\int \psi_{l}(x) d x=1$ for all $l$.

The next lemma is Theorem 1.5.3(i) in [Bo, §1.5].

(3.4.3) Lemma. Let $\psi \in L^{1}\left(\mathbf{R}^{n}\right)$ with $\int \psi(x) d x=1$, and let $\nu$ be a complex measure. Define $d \nu_{l}=\left(\psi_{l} * \nu\right) d x$. Then the sequence of measures $\left\{\nu_{l}\right\}$ is Bernoulli convergent to $\nu$.

The next result follows immediately by applying Lemma (3.4.3) to the bounded continuous function $f(\xi)=e^{-i\langle x, \xi\rangle}$.

(3.4.4) Corollary. Let $\psi \in L^{1}\left(\mathbf{R}^{n}\right)$ with $\int \psi(x) d x=1$, let $\nu$ be a complex measure, and let $\nu_{l}=\psi_{l} * \nu$. Then the Fourier transforms $\hat{\nu}_{l}$ converge pointwise to $\hat{\nu}$.

(3.4.5) Lemma. Let $\lambda, \rho, \mu$, and $\nu$ be complex measures on $\mathbf{R}^{n}$. Then

$$
(\lambda \times \rho) *(\mu \times \nu)=(\lambda * \mu) \times(\rho * \nu) .
$$

Proof. By the definition of convolution and Lemma (2.2.2), we have for all Borel sets $E$ in $\mathbf{R}^{2 n}$,

$$
\begin{aligned}
{[(\lambda \times \rho) *(\mu \times \nu)](E) } \\
\quad=\iint \chi_{E}\left[\left(x_{1}, x_{2}\right)+\left(y_{1}, y_{2}\right)\right] d(\lambda \times \rho)\left(x_{1}, x_{2}\right) d(\mu \times \nu)\left(y_{1}, y_{2}\right) \\
=\iint\left[\iint \chi_{E}\left(x_{1}+y_{1}, x_{2}+y_{2}\right) d \lambda\left(x_{1}\right) d \mu\left(y_{1}\right)\right] d \rho\left(x_{2}\right) d \nu\left(y_{2}\right) \\
\quad=\iint\left[\int \chi_{E}\left(z, x_{2}+y_{2}\right) d(\lambda * \mu)(z)\right] d \rho\left(x_{2}\right) d \nu\left(y_{2}\right) \\
=\int\left[\int \chi_{E}(z, w) d(\lambda * \mu)(z)\right] d(\rho * \nu)(w) \\
=\int \chi_{E} d[(\lambda * \mu) \times(\rho * \nu)] \\
=[(\lambda * \mu) \times(\rho * \nu)](E) .
\end{aligned}
$$


(3.4.6) Lemma. Let $\psi \in L^{1}\left(\mathbf{R}^{n}\right)$ with $\int \psi(x) d x=1$, and let $\mu$, $\nu$ be complex measures on $\mathbf{R}^{n}$. Define $d \mu_{l}=\left(\psi_{l} * \mu\right) d x$ and $d \nu_{l}=\left(\psi_{l} * \nu\right) d x$. Then the sequence of measures $\left\{\mu_{l} \times \nu_{l}\right\}$ is Bernoulli convergent to $\mu \times \nu$.

Proof. Define the function $\Psi$ on $\mathbf{R}^{2 n}$ by $\Psi(x, y)=\psi(x) \psi(y)$. We have then that $\iint|\Psi(x, y)| d x d y<\infty$ and $\iint \Psi(x, y) d x d y=1$, so that by Lemma (3.4.3), the sequence of measures given by $\left[\Psi_{l} *(\mu \times \nu)\right] d x d y$ is Bernoulli convergent to $\mu \times \nu$.

The lemma now follows from the fact that $d\left(\mu_{l} \times \nu_{l}\right)=\left[\Psi_{l} *(\mu \times \nu)\right] d x d y$. To establish this claim, define the measure $\lambda_{l}$ on $\mathbf{R}^{n}$ by $d \lambda_{l}=\psi_{l} d x$. Then $d\left(\lambda_{l} * \mu\right)=\left(\psi_{l} * \mu\right) d x=d \mu_{l}$ and similarly with $\mu$ replaced by $\nu$. Hence by Lemma (3.4.5),

$$
\mu_{l} \times \nu_{l}=\left(\lambda_{l} * \mu\right) \times\left(\lambda_{l} * \nu\right)=\left(\lambda_{l} \times \lambda_{l}\right) *(\mu \times \nu) .
$$

Now consider the measure $\Lambda_{l}$ on $\mathbf{R}^{2 n}$ defined by $d \Lambda_{l}=\Psi_{l} d x d y$. Then $\Lambda_{l}=$ $\lambda_{l} \times \lambda_{l}$ since $\Psi_{l}(x, y)=\psi_{l}(x) \psi_{l}(y)$. But $d\left[\Lambda_{l} *(\mu \times \nu)\right]=\left[\Psi_{l} *(\mu \times \nu)\right] d x d y$, proving the claim and the lemma.

3.5. Generalizing the representation formula. In this section, we obtain a representation theorem analogous to Theorem (3.3.1) for compactly supported measures $\nu_{1}, \nu_{2} \in V^{k}\left(\mathbf{R}^{n}\right)$ that do not necessarily have density functions. Together with Theorem (3.2.1), the following result is Theorem (1.8).

(3.5.1) Theorem. Let $\nu_{1}, \nu_{2} \in V^{k}\left(\mathbf{R}^{n}\right)$ have compact support. If $0<\alpha<2 k+2$ and if $\alpha$ is not even, then

$$
J^{\alpha}\left(\nu_{1}, \nu_{2}\right)=c_{n, \alpha} \int_{\mathbf{R}^{n}} \frac{\hat{\nu}_{1}(x) \overline{\hat{\nu}}_{2}(x)}{|x|^{\alpha+n}} d x,
$$

where $c_{n, \alpha}=2^{-\alpha} \pi^{-n / 2} \Gamma\left(\frac{1}{2}(n+\alpha)\right) / \Gamma\left(-\frac{1}{2} \alpha\right)$.

Proof. Let $\psi$ be a compactly supported $C^{\infty}$ function with $\int \psi(x) d x=1$ and let $\psi_{l}(x)=l^{n} \psi(l x)$. Define $\nu_{j, l}=\psi_{l} * \nu_{j}$. Then $\nu_{j, l}$ is a $C^{\infty}$ function with compact support. Moreover, $\nu_{j, l}$ is in $V^{k}\left(\mathbf{R}^{n}\right)$. By Theorem (3.3.1),

$$
J^{\alpha}\left(\nu_{1, l}, \nu_{2, l}\right)=c_{n, \alpha} \int_{\mathbf{R}^{n}} \frac{\hat{\nu}_{1, l}(x) \overline{\hat{\nu}}_{2, l}(x)}{|x|^{\alpha+n}} d x .
$$

with $c_{n, \alpha}=2^{-\alpha} \pi^{-n / 2} \Gamma\left(\frac{1}{2}(n+\alpha)\right) / \Gamma\left(-\frac{1}{2} \alpha\right)$.

We now discuss the convergence of (3.5.2) as $l \rightarrow \infty$. For the left-hand side of (3.5.2), there exists a single compact set $K$ that contains the support of each $\nu_{1, l} \times \bar{\nu}_{2, l}$ as well as that of $\nu_{1} \times \bar{\nu}_{2}$. Let $\theta(x, y)$ be a compactly supported continuous function on $\mathbf{R}^{2 n}$ that agrees with $|x-y|^{\alpha}$ on $K$. By Lemma (3.4.6), the sequence $\nu_{1, l} \times \bar{\nu}_{2, l}$ is Bernoulli convergent to $\nu_{1} \times \bar{\nu}_{2}$, so that as $l \rightarrow \infty$,

$$
J^{\alpha}\left(\nu_{1, l}, \nu_{2, l}\right)=\int \theta d\left(\nu_{1, l} \times \bar{\nu}_{2, l}\right) \rightarrow \int \theta d\left(\nu_{1} \times \bar{\nu}_{2}\right)=J^{\alpha}\left(\nu_{1}, \nu_{2}\right) .
$$

It remains to treat the right-hand side of (3.5.2). First, we claim that $\hat{\nu}_{1}(x) \overline{\hat{\nu}}_{2}(x) /|x|^{\alpha+n}$ is in $L^{1}\left(\mathbf{R}^{n}\right)$. Indeed, since $\nu_{j}$ is a complex measure, $\hat{\nu}_{j}$ is a bounded continuous function; hence, we need only examine the behavior of the integrand near the origin. But since $\nu_{j}$ has compact support, $\hat{\nu}_{j}$ is in fact $C^{\infty}$ by Lemma (2.3.1). Now the vanishing of $\int x^{\beta} d \nu_{j}(x)$ is equivalent to the 
vanishing of $\partial^{\beta} \hat{\nu}_{j}(0)$; therefore, as in the proof of Theorem (3.3.1), we have by Taylor's formula that $\left|\hat{\nu}_{j}(x)\right| \leq C_{j}|x|^{k+1}$ on $\{x:|x| \leq 1\}$ for some $C_{j}$. Thus,

$$
\frac{\left|\hat{\nu}_{1}(x) \overline{\hat{\nu}}_{2}(x)\right|}{|x|^{\alpha+n}} \leq \frac{C|x|^{2 k+2}}{|x|^{\alpha+n}}, \quad|x| \leq 1,
$$

for some $C$, and so

$$
\int_{\mathbf{R}^{n}} \frac{\left|\hat{\nu}_{1}(x) \hat{\hat{\nu}}_{2}(x)\right|}{|x|^{\alpha+n}} d x<\infty
$$

as claimed.

Second, since $\left\|\hat{\psi}_{l}\right\|_{\infty} \leq\left\|\psi_{l}\right\|_{1}=\|\psi\|_{1}$, we have $\left|\hat{\nu}_{j, l}(x)\right| \leq\|\psi\|_{1}\left|\hat{\nu}_{l}(x)\right|$, and thus

$$
\frac{\left|\hat{\nu}_{1, l}(x) \overline{\hat{\nu}}_{2, l}(x)\right|}{|x|^{\alpha+n}} \leq\|\psi\|_{1}^{2} \frac{\left|\hat{\nu}_{1}(x) \overline{\hat{\nu}}_{2}(x)\right|}{|x|^{\alpha+n}} .
$$

Finally, we have by Corollary (3.4.4) that $\hat{\nu}_{j, l} \rightarrow \hat{\nu}_{j}$ pointwise. Therefore we may apply the Dominated Convergence Theorem to the right hand side of (3.5.2) to obtain

$$
\int_{\mathbf{R}^{n}} \frac{\hat{\nu}_{1, l}(x) \overline{\hat{\nu}}_{2, l}(x)}{|x|^{\alpha+n}} d x \rightarrow \int_{\mathbf{R}^{n}} \frac{\hat{\nu}_{1}(x) \overline{\hat{\nu}}_{2}(x)}{|x|^{\alpha+n}} d x .
$$

3.6. An application to orthonormal bases. Let $q$ be a nonnegative integer and let $2 q<\alpha<2 q+2$. Then by Theorems (3.3.1) and (3.5.1),

$$
(-1)^{q+1} I^{\alpha}(\nu)>0
$$

whenever the nonzero complex measure $\nu \in V^{q}\left(\mathbf{R}^{n}\right)$ either is of the form $d \nu=f d x$ for $f \in \mathscr{S}\left(\mathbf{R}^{n}\right)$ or has compact support. Thus, $(-1)^{q+1} J^{\alpha}$ is an inner product on an appropriate space, and a natural problem to consider is the construction of an orthonormal basis with respect to this inner product. We now treat this problem.

We first describe a Hilbert space $\Omega=\Omega_{n, \alpha}$ which includes all complex measures $\nu \in V^{q}\left(\mathbf{R}^{n}\right)$ of the form $d \nu=f d x$ for $f \in \mathscr{S}\left(\mathbf{R}^{n}\right)$, and all compactly supported complex measures in $V^{q}\left(\mathbf{R}^{n}\right)$.

Let the positive measure $\rho$ on $\mathbf{R}^{n}$ be given by $d \rho=c|x|^{-n-\alpha} d x$ where $c=\left|c_{n, \alpha}\right|$. The mapping $f \rightarrow c^{-1 / 2}|x|^{(n+\alpha) / 2} f$ is a Hilbert space isomorphism between $L^{2}(m)$ and $L^{2}(\rho)$. We claim that any function in $L^{2}(\rho)$ is a tempered function. To justify this claim, first recall that a measurable function $g$ is called tempered if $(1+|x|)^{-N} g \in L^{1}(m)$ for some $N \geq 0$. Note that if $s \geq 0$ (and in particular if $s=(n+\alpha) / 2)$, then

$$
\frac{|x|^{s}}{(1+|x|)^{N}}<\frac{1}{|x|^{N-s}}, \quad|x| \geq 1,
$$

and so $(1+|x|)^{-N}|x|^{s} \in L^{2}(m)$ for $N-s>n / 2$. Therefore by the CauchySchwarz inequality, $(1+|x|)^{-N}|x|^{s} f \in L^{1}(m)$ for $N>s+n / 2$, proving the claim.

Thus, any function in $L^{2}(\rho)$ can be regarded as a tempered distribution. Now the inverse Fourier transform takes $\mathscr{S}^{\prime}\left(\mathbf{R}^{n}\right)$ to $\mathscr{S}^{\prime}\left(\mathbf{R}^{n}\right)$. We may therefore let $\Omega=\mathscr{F}^{-1} L^{2}(\rho)$. The space $\Omega$ is a well-defined set of tempered distributions. 
If $S, T \in \Omega$, then $\hat{S}, \hat{T} \in L^{2}(\rho)$, so we define an inner product $\langle S, T\rangle$ on $\Omega$ to be equal to the inner product $\langle\hat{S}, \hat{T}\rangle$ on $L^{2}(\rho)$. Equipped with this inner product, $\Omega$ becomes a Hilbert space, and the inverse Fourier transform is a Hilbert space isomorphism between $L^{2}(\rho)$ and $\Omega$.

$$
L^{2}(m) \stackrel{\text { mult by } c^{-1 / 2}|x|^{(n+\alpha) / 2}}{\longrightarrow} L^{2}(\rho) \longrightarrow \mathscr{F}^{-1} \longrightarrow \Omega
$$

By Theorems (3.3.1) and (3.5.1), the space $\Omega$ contains the desired measures, and the inner product on $\Omega$ agrees with $(-1)^{q+1} J^{\alpha}$ on these measures. Under these isomorphisms, an orthonormal basis for $L^{2}(m)$ is mapped to an orthonormal basis for $\Omega$.

3.7. More applications. Theorem (3.5.1) can be used to give new proofs of generalizations of properties of $I^{\alpha}$ that appeared in [A3]. Throughout this section, $q$ is a nonnegative integer.

(3.7.1) Proposition. Let $\nu_{1}, \nu_{2}, \ldots, \nu_{m}$ be compactly supported complex measures in $V^{q}\left(\mathbf{R}^{n}\right)$. Suppose $2 q<\alpha<2 q+2$ (so that by Theorem (3.5.1), $(-1)^{q+1} I^{\alpha}\left(\nu_{j}\right)$ is nonnegative). If $z_{1}, z_{2}, \ldots, z_{m}$ are complex numbers with $\sum\left|z_{j}\right|=1$, then

$$
(-1)^{q+1} I^{\alpha}\left(\sum z_{j} \nu_{j}\right) \leq(-1)^{q+1} \sum\left|z_{j}\right| I^{\alpha}\left(\nu_{j}\right) .
$$

Proof. By Theorem (3.5.1),

$$
(-1)^{q+1} I^{\alpha}\left(\sum z_{j} \nu_{j}\right)=\left|c_{n, \alpha}\right| \int_{\mathbf{R}^{n}} \frac{\left|\sum z_{j} \hat{\nu}_{j}(x)\right|^{2}}{|x|^{\alpha+n}} d x .
$$

Now

$$
\left|\sum z_{j} \hat{\nu}_{j}(x)\right|^{2} \leq\left(\sum\left|z_{j}\right|\left|\hat{\nu}_{j}(x)\right|\right)^{2}=\left[\sum\left(\left|z_{j}\right|^{1 / 2}\right)\left(\left|z_{j}\right|^{1 / 2}\left|\hat{\nu}_{j}(x)\right|\right)\right]^{2} .
$$

But by the Cauchy-Schwarz inequality,

$$
\left[\sum\left(\left|z_{j}\right|^{1 / 2}\right)\left(\left|z_{j}\right|^{1 / 2}\left|\hat{\nu}_{j}(x)\right|\right)\right]^{2} \leq\left(\sum\left|z_{j}\right|\right)\left(\sum\left|z_{j}\right|\left|\hat{\nu}_{j}(x)\right|^{2}\right) .
$$

Since $\sum\left|z_{j}\right|=1$, we therefore have

$$
\left|\sum z_{j} \hat{\nu}_{j}(x)\right|^{2} \leq \sum\left|z_{j}\right|\left|\hat{\nu}_{j}(x)\right|^{2}
$$

Thus,

$$
\begin{aligned}
\left|c_{n, \alpha}\right| \int_{\mathbf{R}^{n}} \frac{\left|\sum z_{j} \hat{\nu}_{j}(x)\right|^{2}}{|x|^{\alpha+n}} d x & \leq\left|c_{n, \alpha}\right| \sum\left|z_{j}\right| \int_{\mathbf{R}^{n}} \frac{\left|\hat{\nu}_{j}(x)\right|^{2}}{|x|^{\alpha+n}} d x \\
& =(-1)^{q+1} \sum\left|z_{j}\right| I^{\alpha}\left(\nu_{j}\right) .
\end{aligned}
$$

The following theorem will be used to prove Theorem (4.1.4) and is important in later applications. It follows directly from Theorem (3.5.1).

(3.7.2) Theorem. Let $\nu$ and $\phi$ be compactly supported complex measures on $\mathbf{R}^{n}$ with $\nu \in V^{q}\left(\mathbf{R}^{n}\right)$. If $2 q<\alpha<2 q+2$, then

$$
(-1)^{q+1} I^{\alpha}(\phi * \nu) \leq(-1)^{q+1}\|\phi\|^{2} I^{\alpha}(\nu) .
$$


Proof. By Lemma (2.2.5), $\phi * \nu$ is a compactly supported measure in $V^{q}\left(\mathbf{R}^{n}\right)$. Thus, Theorem (3.5.1) applies and we have

$$
\begin{aligned}
(-1)^{q+1} I^{\alpha}(\phi * \nu) & =\left|c_{n, \alpha}\right| \int_{\mathbf{R}^{n}} \frac{\left.\widehat{\phi * \nu}(x)\right|^{2}}{|x|^{\alpha+n}} d x \\
& =\left|c_{n, \alpha}\right| \int_{\mathbf{R}^{n}} \frac{|\hat{\phi}(x) \hat{\nu}(x)|^{2}}{|x|^{\alpha+n}} d x \\
& \leq\left|c_{n, \alpha}\right|\|\hat{\phi}\|_{\infty}^{2} \int_{\mathbf{R}^{n}} \frac{|\hat{\nu}(x)|^{2}}{|x|^{\alpha+n}} d x \\
& \leq(-1)^{q+1}\|\phi\|^{2} I^{\alpha}(\nu) . \quad
\end{aligned}
$$

\section{FURTHER APPLICATIONS AND EXAMPLES}

4.1. The general method for lower bounds. In this section, we discuss a general method for bounding $-I^{\alpha}$ (or $I^{\alpha}$ ) from below. For the case of real-valued signed measures, these techniques first appeared in [A2] and [A3]. Here we use results from $\S 3$ to extend to the case of complex measures. Throughout this section, $q$ and $k$ will always denote nonnegative integers.

Let $\nu$ be a compactly supported nonzero complex measure in $V^{q}\left(\mathbf{R}^{n}\right)$, and let $2 q<\alpha<2 q+2$. Then we know by Theorem (3.5.1) that $(-1)^{q+1} I^{\alpha}(\nu)>0$. We seek stronger lower bounds for $(-1)^{q+1} I^{\alpha}(\nu)$.

We shall work in $\mathbf{R}^{n+1}$, letting $(x, s)$ denote the typical point of $\mathbf{R}^{n+1}$, with $x=\left(x_{1}, \ldots, x_{n}\right) \in \mathbf{R}^{n}$ and $s \in \mathbf{R}$. Let $P \subset \mathbf{R}^{n+1}$ be the $s=0$ hyperplane in $\mathbf{R}^{n+1}$, and let $L \subset \mathbf{R}^{n+1}$ be the $s$-axis in $\mathbf{R}^{n+1}$ (so that $P$ is identified with $\mathbf{R}^{n}$, and $L$ is identified with $\mathbf{R}$ ).

Any complex measure $\nu$ on $\mathbf{R}^{n}$ gives rise to a complex measure $\nu^{\prime}$ on $\mathbf{R}^{n+1}$ defined by $\nu^{\prime}(E)=\nu(E \cap P)$. Similarly, any complex measure $\phi$ on $\mathbf{R}$ gives rise to a complex measure $\phi^{\prime \prime}$ on $\mathbf{R}^{n+1}$ given by $\phi^{\prime \prime}(E)=\phi(E \cap L)$. Note that $\operatorname{supp}\left(\nu^{\prime}\right) \subset P$ and $\operatorname{supp}\left(\phi^{\prime \prime}\right) \subset L$.

(4.1.1) Lemma. If $\nu \in V^{k}\left(\mathbf{R}^{n}\right)$, then $\nu^{\prime} \in V^{k}\left(\mathbf{R}^{n+1}\right)$.

Proof. We first observe that $\nu \in M^{k}\left(\mathbf{R}^{n}\right)$ implies $\nu^{\prime} \in M^{k}\left(\mathbf{R}^{n+1}\right)$. Indeed, since $\operatorname{supp}\left(\nu^{\prime}\right) \subset P$, we have

$$
\int_{\mathbf{R}^{n+1}}|(x, s)|^{k} d\left|\nu^{\prime}\right|(x, s)=\int_{s=0}|(x, s)|^{k} d\left|\nu^{\prime}\right|(x, s)=\int_{\mathbf{R}^{n}}|x|^{k} d|\nu|(x) .
$$

Now let $\beta=(\gamma, \delta)=\left(\gamma_{1}, \ldots, \gamma_{n}, \delta\right)$ be a multi-index with $|\beta| \leq k$. Then

$$
\int_{\mathbf{R}^{n+1}}(x, s)^{\beta} d \nu^{\prime}(x, s)=\int_{s=0} x^{\gamma} s^{\delta} d \nu^{\prime}(x, s) .
$$

If $\delta>0$, then the integrand vanishes. On the other hand, if $\delta=0$, then $|\gamma|=|\beta| \leq k$, and the integral is zero since $\nu \in V^{k}\left(\mathbf{R}^{n}\right)$.

A similar proof shows that $\phi \in V^{k}(\mathbf{R})$ implies $\phi^{\prime \prime} \in V^{k}\left(\mathbf{R}^{n+1}\right)$.

(4.1.2) Lemma. If $\nu \in M\left(\mathbf{R}^{n}\right)$ and $\phi \in M(\mathbf{R})$, then the measures $\nu^{\prime} * \phi^{\prime \prime}$ and $\nu \times \phi$ on $\mathbf{R}^{n+1}$ are identical.

Proof. By uniqueness, it suffices to show that

$$
\left(\nu^{\prime} * \phi^{\prime \prime}\right)(E \times F)=\nu(E) \phi(F)
$$


for all $E \subset \mathbf{R}^{n}, F \subset \mathbf{R}$. Now by the definition of convolution,

$$
\left(\nu^{\prime} * \phi^{\prime \prime}\right)(E \times F)=\left(\nu^{\prime} \times \phi^{\prime \prime}\right)\left(A^{-1}(E \times F)\right),
$$

where $A: \mathbf{R}^{n+1} \times \mathbf{R}^{n+1} \rightarrow \mathbf{R}^{n+1}$ is the addition operation on $\mathbf{R}^{n+1}$. The lemma follows from the fact that $\mathbf{R}^{n+1}$ is the internal direct sum of $P$ and $L$.

The next result follows immediately from Lemmas (2.2.5), (4.1.1), and (4.1.2).

(4.1.3) Corollary. If $\nu \in V^{q}\left(\mathbf{R}^{n}\right)$ and $\phi \in M^{q}(\mathbf{R})$, then $\nu \times \phi \in V^{q}\left(\mathbf{R}^{n+1}\right)$.

(4.1.4) Theorem. Let $\nu$ be a compactly supported complex measure in $V^{q}\left(\mathbf{R}^{n}\right)$, and let $2 q<\alpha<2 q+2$. Let $\phi$ be a compactly supported complex measure on $\mathbf{R}$. Then

$$
(-1)^{q+1} I^{\alpha}(\nu \times \phi) \leq(-1)^{q+1}\|\phi\|^{2} I^{\alpha}(\nu) .
$$

Proof. Let $\nu^{\prime}$ and $\phi^{\prime \prime}$ be the associated complex measures on $\mathbf{R}^{n+1}$ described above Lemma (4.1.1). By Lemma (4.1.1), $\nu^{\prime} \in V^{q}\left(\mathbf{R}^{n+1}\right)$. Therefore by applying Theorem (3.7.2) to $\nu^{\prime}$ and $\phi^{\prime \prime}$, we obtain

$$
(-1)^{q+1} I^{\alpha}\left(\nu^{\prime} * \phi^{\prime \prime}\right) \leq(-1)^{q+1}\left\|\phi^{\prime \prime}\right\|^{2} I^{\alpha}\left(\nu^{\prime}\right) .
$$

Since $\operatorname{supp}\left(\nu^{\prime}\right) \subset P$ and $\operatorname{supp}\left(\phi^{\prime \prime}\right) \subset L$, we have

$$
I^{\alpha}\left(\nu^{\prime}\right)=\int_{P} \int_{P}|x-y|^{\alpha} d \nu^{\prime}(x) d \bar{\nu}^{\prime}(y)=I^{\alpha}(\nu)
$$

and

$$
\left\|\phi^{\prime \prime}\right\|=\left|\phi^{\prime \prime}\right|(L)=\|\phi\| .
$$

But Lemma (4.1.2) shows that $\nu^{\prime} * \phi^{\prime \prime}$ is equal to $\nu \times \phi$.

The method for bounding $(-1)^{q+1} I^{\alpha}(\nu)$ from below will be to find lower bounds for $(-1)^{q+1} I^{\alpha}(\nu \times \phi)$ and apply Theorem (4.1.4).

The following additional notation will be useful. For $x \in P$, let $L_{x}$ denote the line in $\mathbf{R}^{n+1}$ through $x$ and orthogonal to $P$. If $\phi$ is a complex measure on $\mathbf{R}$, define the measure $\phi_{x}$ by $\phi_{x}(E)=\phi\left(E \cap L_{x}\right)$.

(4.1.5) Theorem. Let $\mu$ and $\nu$ be compactly supported complex measures on $\mathbf{R}^{n}$, and let $\phi$ be a compactly supported complex measures on $\mathbf{R}$. Then

$$
J^{\alpha}(\mu \times \phi, \nu \times \phi)=\int_{\mathbf{R}^{n}} \int_{\mathbf{R}^{n}} J^{\alpha}\left(\phi_{x}, \phi_{y}\right) d \mu(x) d \bar{\nu}(y) .
$$

Proof. Since all measures here have compact support, we may freely apply the Fubini Theorem, so that

$$
J^{\alpha}(\mu \times \phi, \nu \times \phi)=\iint\left[\iint|(x, s)-(y, t)|^{\alpha} d \phi(s) d \bar{\phi}(t)\right] d \mu(x) d \bar{\nu}(y) .
$$

But for fixed $x_{0}, y_{0} \in \mathbf{R}^{n}$,

$$
J^{\alpha}\left(\phi_{x_{0}}, \phi_{y_{0}}\right)=\int_{(y, t)} \int_{(x, s)}|(x, s)-(y, t)|^{\alpha} d \phi_{x_{0}}(x, s) d \bar{\phi}_{y_{0}}(y, t) .
$$

Now since $\phi_{x_{0}}$ has support entirely within the line $L_{x_{0}}$, and $\phi_{y_{0}}$ has support entirely within the line $L_{y_{0}}$, this double integral is equal to

$$
\int_{t} \int_{s}\left|\left(x_{0}, s\right)-\left(y_{0}, t\right)\right|^{\alpha} d \phi(s) d \bar{\phi}(t) \text {. }
$$

The conclusion follows. 
When the measure $\phi$ is understood from context, it will sometimes be convenient to write $J^{\alpha}\left(\phi_{x}, \phi_{y}\right)=J^{\alpha}(x, y)$. Furthermore, the proof of the previous theorem showed that

$$
J^{\alpha}(x, y)=\int_{t} \int_{s}|(x, s)-(y, t)|^{\alpha} d \phi(s) d \bar{\phi}(t) .
$$

Now if $r=|x-y|$, then

$$
|(x, s)-(y, t)|^{\alpha}=\left[(x-y)^{2}+(s-t)^{2}\right]^{\alpha / 2}=\left[r^{2}+(s-t)^{2}\right]^{\alpha / 2},
$$

so that

$$
J^{\alpha}(x, y)=\int_{t} \int_{s}\left[r^{2}+(s-t)^{2}\right]^{\alpha / 2} d \phi(s) d \bar{\phi}(t) .
$$

Hence, $J^{\alpha}(x, y)$ does not depend on the location of the points $x$ and $y$ but only on the distance between them. This discussion motivates the following definition.

(4.1.6) Definition. If $\phi$ is a compactly supported complex measure on $\mathbf{R}$, if $\alpha>0$, and if $r$ is any real number, then let

$$
J^{\alpha}(\phi, r)=\int_{t} \int_{s}\left[r^{2}+(s-t)^{2}\right]^{\alpha / 2} d \phi(s) d \bar{\phi}(t) .
$$

We emphasize that $J^{\alpha}(\phi, r)$ can be considered as a function of $r$ with parameters $\alpha$ and $\phi$, in which case $J^{\alpha}(\phi, r)$ does not depend on the points $x$ and $y$, the dimension $n$ of $\mathbf{R}^{n}$, or the measures $\mu$ and $\nu$ from the foregoing discussion. However, in a particular context, if $r$ is taken to be the distance between the two points $x$ and $y$, then $J^{\alpha}(\phi, r)=J^{\alpha}(x, y)$ and the dependence on $x$ and $y$ is as described earlier.

The following two theorems are proved in [A3].

(4.1.7) Theorem (R. Alexander). Let the nonzero signed atomic measure $\phi \in$ $V^{k}(\mathbf{R})$ have compact support. If $2 q<\alpha<2 q+2$, where $0 \leq q \leq k$, then $(-1)^{q+1} J^{\alpha}(\phi, r)$ is a strictly decreasing positive function of $r$.

(4.1.8) Theorem (R. Alexander). Let the signed atomic measure $\phi \in V^{k}(\mathbf{R})$ have support in the interval $\left[-\frac{1}{2} h, \frac{1}{2} h\right]$ for some $0<h \leq \frac{1}{2}$, and let $\|\phi\|=1$. If $\alpha>0$ and $r \geq 2$, then

$$
\left|J^{\alpha}(\phi, r)\right|<c_{\alpha} h^{2 k} / r^{2 k+2-\alpha},
$$

where $c_{\alpha}$ is a positive constant depending only on $\alpha$. If $0<\alpha<2$, then $c_{\alpha}$ may be replaced by 1 .

We observe that Theorem (4.1.8) is most useful if $\alpha<2 k+2$.

Suppose $\mu$ and $\nu$ are compactly supported complex measures in $V^{q}\left(\mathbf{R}^{n}\right)$, and let $2 q<\alpha<2 q+2$. Suppose we introduce a measure $\phi$ that is a nonzero, signed, compactly supported atomic measure in $V^{k}(\mathbf{R})$ where $k \geq q$. Let $r=|x-y|$. Then by Theorem (4.1.5) we have

$$
(-1)^{q+1} J^{\alpha}(\mu \times \phi, \nu \times \phi)=\int_{\mathbf{R}^{n}} \int_{\mathbf{R}^{n}}\left[(-1)^{q+1} J^{\alpha}(\phi, r)\right] d \mu(x) d \bar{\nu}(y) .
$$


This formula should be compared to the definition of $J^{\alpha}$ :

$$
J^{\alpha}(\mu, \nu)=\int_{\mathbf{R}^{n}} \int_{\mathbf{R}^{n}} r^{\alpha} d \mu(x) d \bar{\nu}(y) .
$$

Notice that in both formulas (4.1.9) and (4.1.10), the integrands are positive. However, the integrand in (4.1.10) is a strictly increasing function of $r$, while the integrand in (4.1.9) is a strictly decreasing function of $r$ by Theorem (4.1.7). In fact, Theorem (4.1.8) shows that the integrand in (4.1.9) decreases faster than $c_{\alpha, k} / r^{2 k+2-\alpha}$ for $r \geq 2$. These properties will be the key to obtaining lower bounds for $(-1)^{q+1} I^{\alpha}(\nu \times \phi)$ and hence $(-1)^{q+1} I^{\alpha}(\nu)$ via Theorem (4.1.4).

We conclude this section by recording some lemmas. The following fact from [A3] guarantees the existence of a measure $\phi$ satisfying the conditions in the previous paragraph.

(4.1.11) Lemma ( $\mathbf{R}$. Alexander). Given $k+2$ distinct points in $\mathbf{R}$, there is a nonzero signed measure $\phi \in V^{k}(\mathbf{R})$ that is supported by these points.

The following lemma will be used in the proof of Theorem (4.2.2). The proof is a direct generalization of the proof of [A2, Lemma 8] to the case of complex $z_{i}$.

(4.1.12) Lemma. Let $M$ be a positive integer, and let $a_{i j} \geq 0$, with $a_{i j}=a_{j i}$, for $i, j \in\{1, \ldots, M\}$. Suppose that for each $i$,

$$
\sum_{j=1}^{M} a_{i j} \leq K,
$$

for some $K$ which is independent of $i$. Then for any complex numbers $z_{1}, \ldots$, $z_{M}$,

$$
\left|\sum_{i, j=1}^{M} a_{i j} z_{i} \bar{z}_{j}\right| \leq K \sum_{i=1}^{M}\left|z_{i}\right|^{2} .
$$

Finally, the proofs of the theorems obtained below for checkerboard and continuous examples will use the notation and results of the following lemma.

(4.1.13) Lemma. Let the signed atomic measure $\phi \in V^{k}(\mathbf{R})$ have support in the interval $\left[-\frac{1}{2} h, \frac{1}{2} h\right]$ for some $0<h \leq \frac{1}{2}$, and let $\|\phi\|=1$. Let $2 q<\alpha<2 q+2$, for some $0 \leq q \leq k$. Fix $x \in \mathbf{R}^{n}$ and let $\delta>0$. Define

$$
c_{1}(\delta)=c_{1}(\delta, n, \alpha, \phi)=\int_{|y-x|<\delta}(-1)^{q+1} J^{\alpha}(x, y) d m(y)
$$

and

$$
c_{2}(\delta)=c_{2}(\delta, n, \alpha, \phi)=\int_{|y-x|>\delta}(-1)^{q+1} J^{\alpha}(x, y) d m(y) .
$$

If $k \geq \frac{1}{2}(n+1)+q$, then $c_{1}(\delta)+c_{2}(\delta) \equiv c_{2}(0)<\infty$, and so $c_{2}(\delta) \rightarrow 0$ as $\delta \rightarrow \infty$.

Proof. Since

$$
(-1)^{q+1} J^{\alpha}(\phi, r)<c_{\alpha} h^{2 k} / r^{2 k+2-\alpha} \text { for } r \geq 2,
$$

and since

$$
0<(-1)^{q+1} J^{\alpha}(\phi, r)<(-1)^{q+1} I^{\alpha}(\phi) \text { for all } r,
$$


we have

$$
\begin{aligned}
c_{2}(0) & =\int_{S^{n-1}} \int_{0}^{\infty}(-1)^{q+1} J^{\alpha}(\phi, r) r^{n-1} d r d \sigma \\
& \leq \sigma\left(S^{n-1}\right)\left[(-1)^{q+1} I^{\alpha}(\phi) \int_{0}^{2} r^{n-1} d r+c_{\alpha} h^{2 k} \int_{2}^{\infty} \frac{r^{n-1}}{r^{2 k+2-\alpha}} d r\right] .
\end{aligned}
$$

But $\alpha<2 q+2$ and $k \geq \frac{1}{2}(n+1)+q$. Therefore,

$$
\int_{2}^{\infty} \frac{r^{n-1}}{r^{2 k+2-\alpha}} d r<\int_{2}^{\infty} \frac{1}{r^{2}} d r<\infty .
$$

It should be observed that by the discussion preceding Definition (4.1.6), the quantities $c_{1}(\delta)$ and $c_{2}(\delta)$ are independent of the choice of the point $x \in \mathbf{R}^{n}$.

4.2. Checkerboard examples. A key feature of the estimates obtained in [A2] and [A3] is that the measures involved have atoms. In particular, the weight of a measure at each of these points is of substantially greater magnitude than in the surrounding neighborhood, and this concentration of weight is used in a decisive manner. We wish to consider nonatomic measures, which do not have such concentrations at points. As a first example of a measure whose weight is smeared out over open sets, we consider a generalized checkerboard measure as discussed in the hypotheses of Theorem (1.1). The following theorem contains Theorem (1.10) when $n=2$.

(4.2.1) Theorem. Suppose the function $f$ on $\mathbf{R}^{n}$ is defined on $N^{n}$ open cubes $S_{i}$, each of side length $s$, arranged in an $N \times \cdots \times N$ lattice. Let $f$ have value $z_{i} \in \mathbf{C}$ on $S_{i}$, and suppose $\sum z_{i}=0$. If $d \nu=f d x$ and $0<\alpha<2$, then

$$
-I^{\alpha}(\nu) \geq c_{n, \alpha} s^{2 n+\alpha}\left(\sum\left|z_{i}\right|^{2}\right)
$$

where $c_{n, \alpha}$ is a positive constant independent of the location of the lattice and of the number, size, and weights of the cubes.

Actually we can prove the same inequality regardless of the arrangement of the cubes. The following theorem contains Theorem (4.2.1) as a special case.

(4.2.2) Theorem. Consider a collection of disjoint open cubes $S_{i}$ in $\mathbf{R}^{n}$, each of side length $s$. Let $f$ have value $z_{i} \in \mathbf{C}$ on $S_{i}$, and 0 elsewhere in $\mathbf{R}^{n}$. Suppose $\sum z_{i}=0$. Define $d \nu=f d x$, so that $\nu \in V^{0}\left(\mathbf{R}^{n}\right)$. Let $0<\alpha<2$. Then

$$
-I^{\alpha}(\nu) \geq c_{n, \alpha} s^{2 n+\alpha}\left(\sum\left|z_{i}\right|^{2}\right)
$$

where $c_{n, \alpha}$ is a positive constant independent of the number, size, location, and weights of the cubes.

Proof. Let $k \geq \frac{1}{2}(n+1)$ and let $\phi$ be a compactly supported signed atomic measure in $V^{k}(\mathbf{R})$ with $\|\phi\|=1$. Then by Theorems (4.1.4) and (4.1.5),

$$
-I^{\alpha}(\nu) \geq-I^{\alpha}(\nu \times \phi)=\iint-J^{\alpha}(x, y) d \nu(x) d \bar{\nu}(y) .
$$


Therefore because of the structure of the measure $\nu$, we have

$$
\begin{aligned}
-I^{\alpha}\left(\nu^{\prime}\right) \geq & \sum_{i}\left(\int_{S_{i}} \int_{S_{i}}-J^{\alpha}(x, y) d y d x\right)\left|z_{i}\right|^{2} \\
& +\sum_{i \neq j}\left(\int_{S_{i}} \int_{S_{j}}-J^{\alpha}(x, y) d y d x\right) z_{i} \bar{z}_{j} \\
\geq & \sum_{i}\left(\int_{S_{i}} \int_{S_{i}}-J^{\alpha}(x, y) d y d x\right)\left|z_{i}\right|^{2} \\
& -\left|\sum_{i \neq j}\left(\int_{S_{i}} \int_{S_{j}}-J^{\alpha}(x, y) d y d x\right) z_{i} \bar{z}_{j}\right| .
\end{aligned}
$$

We shall bound this difference away from zero. To obtain a lower bound for the first term, let $E_{i}$ be the open cube of side length $s-2 \delta$ centered in the cube $S_{i}$, and let $F_{i}=S_{i}-E_{i}$. $(\delta>0$ is fixed but will be chosen later.) Then

$$
\int_{S_{i}} \int_{S_{i}}-J^{\alpha}(x, y) d y d x \geq \int_{x \in E_{i}} \int_{|y-x|<\delta}-J^{\alpha}(x, y) d y d x=(s-2 \delta)^{n} c_{1}(\delta),
$$

and so

$$
\sum_{i}\left(\int_{S_{i}} \int_{S_{i}}-J^{\alpha}(x, y) d y d x\right)\left|z_{i}\right|^{2} \geq(s-2 \delta)^{n} c_{1}(\delta) \sum_{i}\left|z_{i}\right|^{2} .
$$

We now obtain an upper bound for the term

$$
\left|\sum_{i \neq j}\left(\int_{S_{i}} \int_{S_{j}}-J^{\alpha}(x, y) d y d x\right) z_{i} \bar{z}_{j}\right| .
$$

Let

$$
a_{i j}= \begin{cases}\int_{S_{i}} \int_{S_{j}}-J^{\alpha}(x, y) d y d x, & \text { for } i \neq j, \\ 0, & \text { for } i=j .\end{cases}
$$

Notice that $a_{i j} \geq 0$ and $a_{i j}=a_{j i}$. We shall show that for all fixed $i$,

$$
\sum_{j} a_{i j} \leq K
$$

where $K$ is independent of $i$; then Lemma (4.1.12) will give the upper bound

$$
\left|\sum_{i, j} a_{i j} z_{i} \bar{z}_{j}\right| \leq K \sum_{i}\left|z_{i}\right|^{2} .
$$

For $i \neq j$,

$$
a_{i j}=\int_{x \in E_{i}} \int_{y \in S_{j}}-J^{\alpha}(x, y) d y d x+\int_{x \in F_{i}} \int_{y \in S_{j}}-J^{\alpha}(x, y) d y d x .
$$

But

$$
\int_{x \in E_{i}} \int_{y \in S_{j}}-J^{\alpha}(x, y) d y d x \leq \int_{x \in E_{i}} \int_{|y-x|>\delta}-J^{\alpha}(x, y) d y d x=(s-2 \delta)^{n} c_{2}(\delta),
$$


and

$\int_{x \in F_{i}} \int_{y \in S_{j}}-J^{\alpha}(x, y) d y d x \leq \int_{x \in F_{i}} \int_{y}-J^{\alpha}(x, y) d y d x=\left[s^{n}-(s-2 \delta)^{n}\right] c_{2}(0)$.

So we indeed have for $i \neq j$ that

$$
a_{i j} \leq(s-2 \delta)^{n} c_{2}(\delta)+P_{n-1, \delta}(s) c_{2}(0)
$$

where $P_{n-1, \delta}(s)$ is an $(n-1)$ th-degree polynomial in $s$ with coefficients depending on $n, \alpha$, and $\delta$. We could now sum over $j$ for each fixed $i$ in (4.2.6) and obtain a bound $K$ to use in (4.2.5), but such an estimate would not be as strong as we should like. The estimate in (4.2.6) is weak, because it holds for all $i$ and $j$ and must therefore allow for the possibility that $S_{i}$ and $S_{j}$ might be within distance $\delta$ of each other. However, given a fixed $S_{i}$, there is a maximum number of $S_{j}$ which may lie within distance $\delta$ of $S_{i}$. A packing argument given in Lemma (4.2.7) below will show that there exists $M(\delta, n)$ such that this maximum number is $C_{n}$ whenever $s>M(\delta, n)$. For fixed $i$, we therefore consider

$$
\sum_{j} a_{i j}=\sum_{j \in J_{1}} a_{i j}+\sum_{j \in J_{2}} a_{i j}
$$

where $J_{1}=J_{1}(i)$ is the index set of all $j$ such that $S_{j}$ lies within distance $\delta$ of $S_{i}$, and $J_{2}=J_{2}(i)$ is the index set of all other $j$. We use the estimate in (4.2.6) only for $j \in J_{1}$, obtaining

$$
\sum_{j \in J_{1}} a_{i j} \leq C_{n}\left[(s-2 \delta)^{n} c_{2}(\delta)+P_{n-1, \delta}(s) c_{2}(0)\right]
$$

For $j \in J_{2}$, on the other hand, we have

$$
\sum_{j \in J_{2}} a_{i j}=\int_{S_{i}} \int_{\bigcup_{J_{2}} S_{j}}-J^{\alpha}(x, y) d y d x \leq \int_{S_{i}} \int_{|y-x|>\delta}-J^{\alpha}(x, y) d y d x=s^{n} c_{2}(\delta) .
$$

We conclude that

$$
\begin{aligned}
\sum_{j} a_{i j} & \leq C_{n}\left[(s-2 \delta)^{n} c_{2}(\delta)+P_{n-1, \delta}(s) c_{2}(0)\right]+s^{n} c_{2}(\delta) \\
& =\left(C_{n}+1\right) c_{2}(\delta) s^{n}+Q_{n-1, \delta}(s)
\end{aligned}
$$

where $Q_{n-1, \delta}(s)$ is an $(n-1)$ th-degree polynomial in $s$ with coefficients depending on $n, \alpha$, and $\delta$. Therefore by Lemma (4.1.12) we obtain the upper bound

$$
\left|\sum_{i, j} a_{i j} z_{i} \bar{z}_{j}\right| \leq\left[\left(C_{n}+1\right) c_{2}(\delta) s^{n}+Q_{n-1, \delta}(s)\right] \sum_{i}\left|z_{i}\right|^{2}
$$

Combining this with (4.2.3) and (4.2.4), we obtain

$$
-I^{\alpha}(\nu) \geq\left[s^{n} c_{1}(\delta)-\left(C_{n}+1\right) c_{2}(\delta) s^{n}-Q_{n-1, \delta}(s)\right] \sum_{i}\left|z_{i}\right|^{2}
$$

Choose $\delta$ so that $c_{1}(\delta)>\left(C_{n}+1\right) c_{2}(\delta)$. Choose $s_{0}>\max (M(\delta, n), 2 \delta)$ sufficiently large so that the quantity in brackets is greater than 1 when $s$ is replaced by $s_{0}$. Note that $s_{0}$ is completely independent of the measure $\nu$ (but 
does depend on $n$ and $\alpha$ ). We have shown that if $\nu_{0}$ is a measure of this type with support squares of side length $s_{0}$, then

$$
-I^{\alpha}\left(\nu_{0}\right) \geq \sum\left|z_{i}\right|^{2} \text {. }
$$

Now consider the measure $\nu$ with support squares of arbitrary side length $s$. Dilate $\nu$ to obtain a measure $\nu_{0}$ with support squares of side length $s_{0}$. Then by scaling,

$$
-\left(s_{0} / s\right)^{2 n+\alpha} I^{\alpha}(\nu)=-I^{\alpha}\left(\nu_{0}\right) .
$$

We conclude that $-I^{\alpha}(\nu) \geq c s^{2 n+\alpha}\left(\sum\left|z_{i}\right|^{2}\right)$, where $c=s_{0}^{-(2 n+\alpha)}$ is a positive constant independent of the number, size, location, and weights of the support squares. This completes the proof of Theorems (4.2.1) and (4.2.2), with the exception of the packing argument given in the following lemma.

(4.2.7) Lemma. Let $N(s, \delta, n)$ be the maximum number of cubes of side length $s$ in $\mathbf{R}^{n}$ that have points within distance $\delta$ of another cube of side length $s$, where all cubes are pairwise disjoint. Then there exist $C_{n}$ and $M(\delta, n)$ such that $N(s, \delta, n) \leq C_{n}$ whenever $s>M(\delta, n)$.

Proof. The diameter of each of these cubes is $\sqrt{n} s$. Let $Q_{s}$ be a cube of side length $s$ in $\mathbf{R}^{n}$ with center at the origin. Any cube having points within distance $\delta$ of the cube $Q_{s}$ must lie completely within distance $\frac{1}{2} \sqrt{n} s+\delta+\sqrt{n} s$ of the origin. Therefore, since there is no overlapping of cubes, we have that the volume of $N(s, \delta, n)+1$ cubes of side length $s$ must be no greater than the volume of the ball of radius $\frac{3}{2} \sqrt{n} s+\delta$; that is,

$$
(N(s, \delta, n)+1) s^{n} \leq \omega_{n}\left(\frac{3}{2} \sqrt{n} s+\delta\right)^{n},
$$

where $\omega_{n}$ is the volume of the ball of unit radius in $\mathbf{R}^{n}$. Thus,

$$
N(s, \delta, n)+1 \leq \omega_{n}\left(\frac{3}{2} \sqrt{n}+s^{-1} \delta\right)^{n} .
$$

Now

$$
\left(\frac{3}{2} \sqrt{n}+s^{-1} \delta\right)^{n}=\left(\frac{3}{2} \sqrt{n}\right)^{n}+s^{-1} \delta P\left(s^{-1} \delta\right),
$$

where $P$ is an $(n-1)$ th-degree polynomial with coefficients depending on $n$. But $\varepsilon P(\varepsilon) \rightarrow 0$ as $\varepsilon \rightarrow 0$, so for each $\delta>0$ there exists $M(\delta, n)$ such that $s^{-1} \delta P\left(s^{-1} \delta\right)<\omega_{n}^{-1}$ whenever $s>M(\delta, n)$. We conclude that $N(s, \delta, n)<$ $\omega_{n}\left(\frac{3}{2} \sqrt{n}\right)^{n}$ for $s>M(\delta, n)$, proving the lemma, and hence Theorems (4.2.1) and (4.2.2).

The following theorem shows the sense in which the inequality of Theorem (4.2.1) is best possible.

(4.2.8) Theorem. Let $s, A>0$, let $N$ be a positive integer, and let $0<\alpha<2$. Then there exist $(2 N)^{n}$ open cubes $S_{i}$ in $\mathbf{R}^{n}$, each of side length $s$, arranged in a $2 N \times \cdots \times 2 N$ lattice, along with $(2 N)^{n}$ numbers $z_{i}$ satisfying $\sum z_{i}=0$ and $\sum\left|z_{i}\right|^{2}=A$, such that if $f$ is defined to have value $z_{i}$ on $S_{i}$ and if $d \nu=f d x$, then

$$
-I^{\alpha}(\nu) \leq C_{n, \alpha} s^{2 n+\alpha}\left(\sum\left|z_{i}\right|^{2}\right) .
$$

Here $C_{n, \alpha}$ is a positive constant independent of $s, A$, and $N$.

Proof. We shall say that a lattice (of congruent cubes in $\mathbf{R}^{n}$ ) is in standard position if one corner of the lattice is at the origin and if the edges adjacent to this corner lie on the positive coordinate axes. 
First consider the $2 \times \cdots \times 2$ lattice, in standard position, of cubes each of side length 1 . Define a fixed function $g_{0}$ on this lattice by letting $g_{0}$ have value 1 on $\frac{1}{2}\left(2^{n}\right)$ of these cubes and -1 on the remaining $\frac{1}{2}\left(2^{n}\right)$ cubes.

Now for given $s, A$, and $N$, consider the $N \times \cdots \times N$ lattice, in standard position, of $M=N^{n}$ cubes $Q_{k}$, each of side length $2 s$. Each $2 s$-cube contains a smaller $2 \times \cdots \times 2$ lattice of $2^{n}$ cubes, each of side length $s$. One of these smaller $2 \times \cdots \times 2$ lattices of $s$-cubes is in standard position. Define a fixed function $g$ on this smaller lattice by letting $g(x)=w g_{0}\left(s^{-1} x\right)$, where $w=$ $A^{1 / 2}(2 N)^{-n / 2}$. (Thus, $g$ is a dilation and multiple of the function $g_{0}$.)

Let us say that a function $f$ is $g$-distinguished if $f$ is supported by the larger $N \times \cdots \times N$ lattice of cubes $Q_{k}$ and if each restriction $f_{k}$ to $Q_{k}$ is a translation of the function $g$ or $-g$. We shall consider functions $f$ which are $g$-distinguished, and we shall show that one of these functions will suffice.

Notice that a $g$-distinguished function $f$ is supported by a $2 N \times \cdots \times 2 N$ lattice of cubes $S_{i}$ each of side length $s$, and if $z_{i}= \pm w$ is the value of $f$ on $S_{i}$, then $\sum\left|z_{i}\right|^{2}=(2 N)^{n} w^{2}=A$. Also, if $f$ is $g$-distinguished and if we let $d \nu_{k}=f_{k} d x$, then $\nu_{k}\left(Q_{k}\right)=0$ and so $\nu=\sum_{k=1}^{M} \nu_{k}$ has total mass zero.

We further observe that there are two possible choices for each $f_{k}$ (since $f_{k}$ is a translation of either $g$ or $-g$ ), so there are $2^{M}$ possible choices for $f$.

We now consider a probability space where the sample space consists of the $2^{M}$ possible $g$-distinguished functions, giving equal probability to each function. Since

$$
I^{\alpha}(\nu)=\sum_{k} I^{\alpha}\left(\nu_{k}\right)+\sum_{k \neq l} J^{\alpha}\left(\nu_{k}, \nu_{l}\right),
$$

the expected value of $I^{\alpha}(\nu)$ is

$$
E I^{\alpha}(\nu)=\sum_{k} E I^{\alpha}\left(\nu_{k}\right)+\sum_{k \neq l} E J^{\alpha}\left(\nu_{k}, \nu_{l}\right) .
$$

But the expectation $E J^{\alpha}\left(\nu_{k}, \nu_{l}\right)$ is equal to zero for $k \neq l$. Indeed, there are four equally likely outcomes for $J^{\alpha}\left(\nu_{k}, \nu_{l}\right)$, namely

$$
J^{\alpha}\left(\nu_{k}, \nu_{l}\right)= \begin{cases}c_{k, l}, & \text { if } f_{k} \sim g, f_{l} \sim g, \\ -c_{k, l}, & \text { if } f_{k} \sim g, f_{l} \sim-g, \\ -c_{k, l}, & \text { if } f_{k} \sim-g, f_{l} \sim g, \\ c_{k, l}, & \text { if } f_{k} \sim-g, f_{l} \sim-g .\end{cases}
$$

(Here $f_{k} \sim g$ means that $f_{k}$ is a translation of $g$.) Thus, $E J^{\alpha}\left(\nu_{k}, \nu_{l}\right)=0$, and so

$$
E I^{\alpha}(\nu)=\sum_{k} E I^{\alpha}\left(\nu_{k}\right)
$$

If $d \nu_{0}=g_{0} d x$, then by translation invariance and scaling, we have

$$
I^{\alpha}\left(\nu_{k}\right)=I^{\alpha}\left(\nu_{0}\right) s^{2 n+\alpha} w^{2} .
$$

Thus,

$$
E I^{\alpha}(\nu)=\sum_{k} E I^{\alpha}\left(\nu_{k}\right)=\sum_{k} I^{\alpha}\left(\nu_{0}\right) s^{2 n+\alpha} w^{2}=I^{\alpha}\left(\nu_{0}\right) s^{2 n+\alpha} w^{2} M,
$$

or since $w^{2}=A(2 N)^{-n}=A 2^{-n} M^{-1}$,

$$
E\left[-I^{\alpha}(\nu)\right]=-I^{\alpha}\left(\nu_{0}\right) 2^{-n} s^{2 n+\alpha} A .
$$


Therefore, there exists a $g$-distinguished function $f$ with value on each smaller cube $S_{i}$ equal to $z_{i}=w$ or $-w$ as appropriate such that $\sum\left|z_{i}\right|^{2}=(2 N)^{n} w^{2}=$ $A$ and

$$
-I^{\alpha}(\nu)=-I^{\alpha}\left(\nu_{0}\right) 2^{-n} s^{2 n+\alpha} A .
$$

Letting $C_{n, \alpha}=-I^{\alpha}\left(\nu_{0}\right) 2^{-n}$ proves the theorem.

\subsection{Continuous examples.}

(4.3.1) Lemma. Suppose $f$ is a real-valued continuous function on $\mathbf{R}^{n}$ with compact support, and suppose $\int f(x) d x=0$ but $f \not \equiv 0$. Define $\nu \in V^{0}\left(\mathbf{R}^{n}\right)$ by $d \nu=f d x$, and let $0<\alpha<2$. If $x_{0}$ is a point where $\left|f\left(x_{0}\right)\right|=\|f\|_{\infty}$, and if $\varepsilon>0$ is such that $\left|f(x)-f\left(x_{0}\right)\right|<\frac{1}{2}|| f \|_{\infty}$ whenever $\left|x-x_{0}\right|<\varepsilon$, then

$$
-I^{\alpha}(\nu) \geq c_{n, \alpha}\|f\|_{\infty}^{2} \varepsilon^{2 n+\alpha}
$$

where $c_{n, \alpha}$ is a positive constant independent of $f$.

Proof. By translation, we may assume that $x_{0}=0$. Since $I^{\alpha}(\nu)=I^{\alpha}(-\nu)$, we may also assume that $f(0)=\|f\|_{\infty}$. Fix $\delta>0$. $(\delta$ will be chosen later and will depend on $n$ and $\alpha$, but will be independent of $f$.) Consider the dilation $f_{(s)}$ of the function $f$ by a factor of $s>\varepsilon^{-1} \delta$ given by

$$
f_{(s)}(x)=f\left(s^{-1} x\right) \text {. }
$$

Let $f_{1}$ be the restriction of $f_{(s)}$ to the open ball $\{x:|x|<s \varepsilon\}$ and let $f_{2}=$ $f_{(s)}-f_{1}$. Notice that

$$
f_{1}(x) \geq \frac{1}{2}\|f\|_{\infty}, \quad|x|<s \varepsilon .
$$

Define $d \nu_{(s)}=f_{(s)} d x$, and $d \nu_{j}=f_{j} d x$, so that $\nu_{(s)}=\nu_{1}+\nu_{2}$, where $\nu_{1}$ is a positive measure supported by the open ball $\{x:|x|<s \varepsilon\}$ and $\nu_{2}$ is null there. Let $k \geq \frac{1}{2}(n+1)$ and let $\phi$ be a compactly supported signed atomic measure in $V^{k}(\mathbf{R})$ with $\|\phi\|=1$. Then

$$
\nu_{(s)} \times \phi=\nu_{1} \times \phi+\nu_{2} \times \phi,
$$

and by Lemma (2.2.5), the measure $\lambda_{j}=\nu_{j} \times \phi$ is also in $V^{k}\left(\mathbf{R}^{n+1}\right)$. By Theorem (4.1.4),

$$
\begin{aligned}
-I^{\alpha}\left(\nu_{(s)}\right) & \geq-I^{\alpha}\left(\nu_{(s)} \times \phi\right)=-I^{\alpha}\left(\lambda_{1}\right)-2 J^{\alpha}\left(\lambda_{1}, \lambda_{2}\right)-I^{\alpha}\left(\lambda_{2}\right) \\
& \geq-I^{\alpha}\left(\lambda_{1}\right)-2 J^{\alpha}\left(\lambda_{1}, \lambda_{2}\right)
\end{aligned}
$$

since $-I^{\alpha}\left(\lambda_{2}\right) \geq 0$. We have therefore that

$$
-I^{\alpha}\left(\nu_{(s)}\right) \geq-I^{\alpha}\left(\lambda_{1}\right)-2\left|J^{\alpha}\left(\lambda_{1}, \lambda_{2}\right)\right| \text {. }
$$

We shall obtain a lower bound for this difference. We have

$$
-I^{\alpha}\left(\lambda_{1}\right)=\int_{\mathbf{R}^{n}}\left[\int_{\mathbf{R}^{n}}-J^{\alpha}(x, y) d \nu_{1}(y)\right] d \nu_{1}(x) .
$$

Now since $\nu_{1}$ is a positive measure by construction,

$$
-I^{\alpha}\left(\lambda_{1}\right) \geq \int_{|x|<s \varepsilon-\delta}\left[\int_{|y-x|<\delta}-J^{\alpha}(x, y) f_{1}(y) d y\right] f_{1}(x) d x
$$


In this range of integration, $f_{1}(y)$ and $f_{1}(x)$ are bounded below by $\frac{1}{2}\|f\|_{\infty}$. We conclude that

$$
-I^{\alpha}\left(\lambda_{1}\right) \geq \frac{1}{4}\|f\|_{\infty}^{2} c_{1}(\delta) \omega_{n}(s \varepsilon-\delta)^{n}
$$

where $\omega_{n}$ is the volume of the ball of unit radius in $\mathbf{R}^{n}$.

We now obtain an upper bound for the term $\left|J^{\alpha}\left(\lambda_{1}, \lambda_{2}\right)\right|$. We have

$$
\begin{aligned}
\left|J^{\alpha}\left(\lambda_{1}, \lambda_{2}\right)\right| & =\left|\int\left[\int J^{\alpha}(x, y) d \nu_{2}(y)\right] d \nu_{1}(x)\right| \\
& \leq \int\left[\int-J^{\alpha}(x, y) d\left|\nu_{2}\right|(y)\right] d \nu_{1}(x) .
\end{aligned}
$$

Now $\nu_{1}$ is supported by the ball $\{x:|x|<s \varepsilon\}$. Partitioning this ball into two regions, we obtain

$$
\begin{aligned}
\left|J^{\alpha}\left(\lambda_{1}, \lambda_{2}\right)\right| \leq & \int_{|x|<s \varepsilon-\delta}\left[\int-J^{\alpha}(x, y) d\left|\nu_{2}\right|(y)\right] d \nu_{1}(x) \\
& +\int_{s \varepsilon-\delta \leq|x|<s \varepsilon}\left[\int-J^{\alpha}(x, y) d\left|\nu_{2}\right|(y)\right] d \nu_{1}(x) .
\end{aligned}
$$

But $\nu_{2}$ is supported outside the ball $\{y:|y|<s \varepsilon\}$, so

$$
\int-J^{\alpha}(x, y) d\left|\nu_{2}\right|(y) \leq\|f\|_{\infty} \int_{|y| \geq s \varepsilon}-J^{\alpha}(x, y) d y .
$$

If $|x|<s \varepsilon-\delta$, then

$$
\int_{|y| \geq s \varepsilon}-J^{\alpha}(x, y) d y \leq \int_{|y-x| \geq \delta}-J^{\alpha}(x, y) d y=c_{2}(\delta),
$$

while if $s \varepsilon-\delta \leq|x|<s \varepsilon$, then

$$
\int_{|y| \geq s \varepsilon}-J^{\alpha}(x, y) d y \leq \int_{\mathbf{R}^{n}}-J^{\alpha}(x, y) d y=c_{2}(0)
$$

It follows that

$$
\left|J^{\alpha}\left(\lambda_{1}, \lambda_{2}\right)\right| \leq\|f\|_{\infty} c_{2}(\delta) \int_{|x|<s \varepsilon-\delta} d \nu_{1}(x)+\|f\|_{\infty} c_{2}(0) \int_{s \varepsilon-\delta \leq|x|<s \varepsilon} d \nu_{1}(x)
$$

Since

$$
\int_{|x|<s \varepsilon-\delta} d \nu_{1}(x)=\int_{|x|<s \varepsilon-\delta} f_{1}(x) d x=\|f\|_{\infty} \omega_{n}(s \varepsilon-\delta)^{n}
$$

and

$$
\int_{s \varepsilon-\delta \leq|x|<s \varepsilon} d \nu_{1}(x)=\int_{s \varepsilon-\delta \leq|x|<s \varepsilon} f_{1}(x) d x \leq\|f\|_{\infty}\left[\omega_{n}(s \varepsilon)^{n}-\omega_{n}(s \varepsilon-\delta)^{n}\right],
$$

we conclude that

$$
\left|J^{\alpha}\left(\lambda_{1}, \lambda_{2}\right)\right| \leq\|f\|_{\infty}^{2} \omega_{n}\left\{c_{2}(\delta)(s \varepsilon-\delta)^{n}+c_{2}(0)\left[(s \varepsilon)^{n}-(s \varepsilon-\delta)^{n}\right]\right\} .
$$

Combining this with (4.3.2) and (4.3.3), we obtain the desired lower bound (4.3.4)

$$
-I^{\alpha}\left(\nu_{(s)}\right) \geq\|f\|_{\infty}^{2} \omega_{n}\left\{\left[\frac{1}{4} c_{1}(\delta)-2 c_{2}(\delta)\right](s \varepsilon-\delta)^{n}-2 c_{2}(0)\left[(s \varepsilon)^{n}-(s \varepsilon-\delta)^{n}\right]\right\} .
$$


Choose $\delta>0$ such that $\frac{1}{4} c_{1}(\delta)-2 c_{2}(\delta)$ is positive. Note that $\delta$ depends on $n$ and $\alpha$, but not on $f$ (or $s$ or $\varepsilon$ ). Now $(s \varepsilon-\delta)^{n}$ is an $n$ th-degree polynomial in $s \varepsilon$, and $(s \varepsilon)^{n}-(s \varepsilon-\delta)^{n}$ is an $(n-1)$ th-degree polynomial in $s \varepsilon$ (with coefficients depending on $n$ and $\alpha$ ). Since $\frac{1}{4} c_{1}(\delta)-2 c_{2}(\delta)>0$, there exists $C_{n, \alpha}>0$ such that the quantity in braces in (4.3.4) is greater than 1 whenever $s \varepsilon \geq C_{n, \alpha}$. Increase $C_{n, \alpha}$ if necessary so that $C_{n, \alpha}>\delta$. If we take $s_{0}=\varepsilon^{-1} C_{n, \alpha}$, then the measure $\nu_{\left(s_{0}\right)}$ satisfies

$$
-I^{\alpha}\left(\nu_{\left(s_{0}\right)}\right) \geq \omega_{n}\|f\|_{\infty}^{2}
$$

Finally, by scaling,

$$
-I^{\alpha}(\nu)=-\frac{1}{s_{0}^{2 n+\alpha}} I^{\alpha}\left(\nu_{\left(s_{0}\right)}\right) \geq\left(\frac{\varepsilon}{C_{n, \alpha}}\right)^{2 n+\alpha} \omega_{n}\|f\|_{\infty}^{2}=c_{n, \alpha} \varepsilon^{2 n+\alpha}\|f\|_{\infty}^{2} .
$$

The following theorem contains Theorem (1.11) when $n=2$.

(4.3.5) Theorem. Suppose that the real-valued, compactly supported, continuously differentiable function $f$ on $\mathbf{R}^{n}$ satisfies $\int f(x) d x=0$ but $f \not \equiv 0$. Define $\nu \in V^{0}\left(\mathbf{R}^{n}\right)$ by $\nu(E)=\int_{E} f(x) d x$ and let $0<\alpha<2$. Then

$$
-I^{\alpha}(\nu) \geq c_{n, \alpha}\||\operatorname{grad} f|\|_{\infty}^{-2 n-\alpha}\|f\|_{\infty}^{2 n+\alpha+2},
$$

where $c_{n, \alpha}$ is a positive constant independent of $f$.

Proof. Select a point $x_{0}$ where $\left|f\left(x_{0}\right)\right|=\|f\|_{\infty}$, and since $I^{\alpha}(\nu)=I^{\alpha}(-\nu)$, we may assume that $f\left(x_{0}\right)=\|f\|_{\infty}$. Letting $S=\||\operatorname{grad} f|\|_{\infty}$, we have that the absolute value of any directional derivative of $f$ is never greater than $c_{n} S$, where $c_{n}$ is a dimensional constant. Thus, we must have $f(x) \geq \psi(x)$ for $\left|x-x_{0}\right| \leq 2 \varepsilon$, where $2 \varepsilon=c_{n}^{-1} S^{-1} M$ and $\psi$ is the "cone" function defined by

$$
\psi(x)=-(M / 2 \varepsilon)\left|x-x_{0}\right|+M, \quad\left|x-x_{0}\right| \leq 2 \varepsilon .
$$

This choice of $\varepsilon$ will satisfy the hypotheses of the previous lemma, and the theorem follows immediately.

The following shows the sense in which the inequality of Theorem (4.3.5) is best possible.

(4.3.6) Theorem. Let $M, S>0$. Then there exists a real-valued, compactly supported, continuously differentiable function $f$ on $\mathbf{R}^{n}$ with $\int f(x) d x=0$ having $\|f\|_{\infty}=M$ and $\||\operatorname{grad} f|\|_{\infty}=S$ such that if $d \nu=f d x$ and $0<\alpha<$ 2 , then

$$
-I^{\alpha}(\nu) \leq C_{n, \alpha}\||\operatorname{grad} f|\|_{\infty}^{-2 n-\alpha}\|f\|_{\infty}^{2 n+\alpha+2} .
$$

Here $C_{n, \alpha}$ is a positive constant independent of $M$ and $S$.

Proof. Let $x_{1}$ and $x_{2}$ be points in $\mathbf{R}^{n}$ at distance $2.4 \varepsilon$ from each other. (The number $\varepsilon$ will be chosen later and will depend on $M$ and $S$.) Define the function $r_{j}$ on $\mathbf{R}^{n}$ by letting $r_{j}(x)=\left|x-x_{j}\right|$. Define a continuous, even, piecewise linear "truncated cone" function $\psi$ on $\mathbf{R}$ by setting

$$
\psi(r)= \begin{cases}M, & \text { for }|r| \leq 0.1 \varepsilon \\ 0, & \text { for }|r| \geq 1.1 \varepsilon\end{cases}
$$

and requiring $\psi$ to be linear with slope $\varepsilon^{-1} M$ on the intervals $0.1 \varepsilon \leq|r| \leq$ $1.1 \varepsilon$. 
Define $g_{j}$ on $\mathbf{R}^{n}$ by $g_{j}(x)=\psi\left(r_{j}(x)\right)$, so $g_{j}$ is supported by $B\left(x_{j}, 1.1 \varepsilon\right)$. Let $g=g_{1}-g_{2}$. Note $|\operatorname{grad} g|$ is constant on $\bigcup_{j}\left[B\left(x_{j}, 1.1 \varepsilon\right)-B\left(x_{j}, 0.1 \varepsilon\right)\right]$. This constant value will be $S$ if $\varepsilon$ is taken to be $c_{n} M S^{-1}$, where $c_{n}$ is a dimensional constant.

We now smooth out $g$ on $B\left(x_{j}, 0.2 \varepsilon\right)$ and $B\left(x_{j}, 1.2 \varepsilon\right)-B\left(x_{j}, \varepsilon\right)$. Specifically, define positive $C^{1}$ functions $f_{j}$ on $\mathbf{R}^{n}$ such that

(a) $f_{j}$ is supported by $B\left(x_{j}, 1.2 \varepsilon\right)$,

(b) $f_{j}$ agrees with $g_{j}$ on $B\left(x_{j}, 1.1 \varepsilon\right)-B\left(x_{j}, 0.2 \varepsilon\right)$,

(c) the function $f=f_{1}-f_{2}$ is $C^{1}$,

(d) $\|\mid \operatorname{grad} f\|_{\infty}=S$,

(e) $\|f\|_{\infty}=M$.

Now let $d \nu_{j}=f_{j} d x$. Then $\nu=\nu_{1}-\nu_{2}$ and so

$$
I^{\alpha}(\nu)=I^{\alpha}\left(\nu_{1}\right)-2 J^{\alpha}\left(\nu_{1}, \nu_{2}\right)+I^{\alpha}\left(\nu_{2}\right) .
$$

But $\nu_{j}$ is a positive measure, so $I^{\alpha}\left(\nu_{j}\right)>0$. Thus, $-I^{\alpha}(\nu)<2 J^{\alpha}\left(\nu_{1}, \nu_{2}\right)$. Using the fact that $\nu_{1}$ and $\nu_{2}$ are supported by tangent balls each of radius $1.2 \varepsilon$, we make the computation

$$
\begin{aligned}
J^{\alpha}\left(\nu_{1}, \nu_{2}\right) & =\iint|x-y|^{\alpha} d \nu_{1}(x) d \nu_{2}(y) \leq[4(1.2 \varepsilon)]^{\alpha} \int d \nu_{1} \int d \nu_{2} \\
& =(4.8 \varepsilon)^{\alpha} \int f_{1} \int f_{2} \leq(4.8 \varepsilon)^{\alpha}\left[\omega_{n}(1.2 \varepsilon)^{n} M\right]^{2} \\
& =(4.8)^{\alpha}(1.2)^{2 n} \omega_{n}^{2} \varepsilon^{2 n+\alpha} M^{2} .
\end{aligned}
$$

Since $\varepsilon=c_{n} M S^{-1}$, we conclude that

$$
-I^{\alpha}(\nu)<C_{n, \alpha} S^{-2 n-\alpha} M^{2 n+\alpha+2} .
$$

4.4. Integral geometric notions. In this section we discuss the motion-invariant measure on sets of hyperplanes, and we generalize some of the integral geometric results of [A1, A2, and A3] to complex measures.

Let $\mathbf{P}^{n}$ be the set of all hyperplanes in $\mathbf{R}^{n}$. Each hyperplane $h \in \mathbf{P}^{n}$ can be written $h=\left\{x \in \mathbf{R}^{n}:\langle x, \sigma\rangle=t\right\}$, where $\sigma \in S^{n-1}$ is a unit vector in $\mathbf{R}^{n}$ and $t \in \mathbf{R}$. Since $(\sigma, t)$ and $(-\sigma,-t)$ determine the same hyperplane, there is a two-to-one correspondence between $S^{n-1} \times \mathbf{R}$ and $\mathbf{P}^{n}$. Endow $\mathbf{P}^{n}$ with the topological structure induced by this correspondence.

The product of Euclidean surface measure on $S^{n-1}$ and Lebesgue measure on $\mathbf{R}$ induces a motion-invariant Borel measure on $\mathbf{P}^{n}$ that is unique up to multiplication by a constant. This measure can also be realized as follows. Let $l(\sigma)$ be the directed line through the origin containing the unit vector $\sigma$. For a collection $T$ of hyperplanes, let $T(\sigma)$ denote the collection of hyperplanes in $T$ which are orthogonal to $\sigma$. Set $\rho(\sigma)=m(T(\sigma) \cap l(\sigma))$, where $m$ is onedimensional Lebesgue measure on $l(\sigma)$. Define $\eta_{n}(T)=c_{n} \int \rho(\sigma) d \sigma$, where $d \sigma$ denotes integration with respect to Euclidean surface measure on the unit sphere $S^{n-1}$, and $c_{n}$ is a suitably chosen constant.

The following lemma collects some relevant facts about $\eta_{n}$. A more detailed discussion of these integral geometric notions can be found in [Sa]. 
(4.4.1) Lemma. The measure $\eta_{n}$ is a motion-invariant Borel measure on $\mathbf{P}^{n}$ satisfying the following:

(a) For any compact set $K$ in $\mathbf{R}^{n}$, the collection of hyperplanes intersecting the convex hull of $K$ has finite $\eta_{n}$-measure.

(b) For $x \in \mathbf{R}^{n}, \eta_{n}\{h: x \in h\}=0$.

(c) For $x, y \in \mathbf{R}^{n}, \eta_{n}\{h: h \cap \overline{x y} \neq \varnothing\}=C_{n}|x-y|$, where $C_{n}$ is a dimensional constant which depends on the choice of $c_{n}$ in the definition of $\eta_{n}$.

In light of property $(c)$ in the preceding lemma, from now on we shall assume that $\eta_{n}$ is normalized so that

$$
|x-y|=\frac{1}{2} \eta_{n}\{h: h \cap \overline{x y} \neq \varnothing\} .
$$

We remark that this is not the usual normalization for the motion-invariant measure on hyperplanes, but it will be the most useful for our purposes. Under this normalization, the measure $\eta_{n}$ is induced from $\eta_{n+1}$ in a natural way. Explicitly, identify $\mathbf{R}^{n}$ with $P$, the $s=0$ hyperplane of $\mathbf{R}^{n+1}$, as in $\S 4.1$. If $T$ is a collection of $(n-1)$-planes in $\mathbf{R}^{n}$, then let $T^{\prime}$ be the collection of all $n$ planes in $\mathbf{R}^{n+1}$ whose intersections with $P$ are in $T$. Then $\eta_{n+1}\left(T^{\prime}\right)=\eta_{n}(T)$. We shall often drop the explicit reference to the dimension and refer simply to $\eta$.

The next lemma follows from Lemma (4.4.1b), as shown in [A1, Lemma 3.1]. (4.4.3) Lemma. Let $\nu$ be a complex measure on $\mathbf{R}^{n}$ and let $E=\{h:|\nu|(h) \neq$ $0\}$. Then $\eta(E)=0$.

We shall also use oriented hyperplanes in $\mathbf{R}^{n}$. If we associate the pair $(\sigma, t)$ with the open half-space $H^{+}=H^{+}(\sigma, t)=\left\{x \in \mathbf{R}^{n}:\langle x, \sigma\rangle<t\right\}$, then different open half-spaces are determined by $(\sigma, t)$ and $(-\sigma,-t)$. Thus, $S^{n-1} \times \mathbf{R}$ can be identified with the space of oriented hyperplanes in $\mathbf{R}^{n}$. Let $H^{-}=$ $H^{-}(\sigma, t)=\left\{x \in \mathbf{R}^{n}:\langle x, \sigma\rangle>t\right\}$.

With proper normalization, the motion-invariant measure on sets of oriented hyperplanes in $\mathbf{R}^{n}$ can also be denoted by $\eta_{n}$, or simply $\eta$, and equation (4.4.2) will be valid whether the set $\{h: h \cap \overline{x y} \neq \varnothing\}$ is assumed to be a set of oriented or nonoriented hyperplanes.

The next two theorems contain the appropriate generalizations of Alexander's formulas (1.4) and (1.5) to the case of complex measures.

(4.4.4) Theorem. Suppose $\mu, \nu \in M^{1}\left(\mathbf{R}^{n}\right)$. Then

(a) $J^{1}(\mu, \nu)=\frac{1}{2}\left[\int \mu\left(H^{+}\right) \overline{\nu\left(H^{-}\right)} d \eta(h)+\int \mu\left(H^{-}\right) \overline{\nu\left(H^{+}\right)} d \eta(h)\right]$,

(b) $I^{1}(\nu)=\int \operatorname{Re}\left[\nu\left(H^{+}\right) \overline{\nu\left(H^{-}\right)}\right] d \eta(h)$.

Proof. For $x, y \in \mathbf{R}^{n}$ and $h$ an oriented hyperplane in $\mathbf{R}^{n}$, define $\chi(x, y, h)$ $=1$ if $h$ intersects the open segment $\overline{x y}$ in exactly one point, and $\chi(x, y, h)$ $=0$ otherwise. Then by (4.4.2)

$$
|x-y|=\frac{1}{2} \int \chi(x, y, h) d \eta(h),
$$

so that

$$
\int \chi d(|\mu| \times|\nu| \times \eta)=2 \iint|x-y| d|\mu|(x) d|\nu|(y)=2 J^{1}(|\mu|,|\nu|),
$$


and this is finite since $\mu, \nu \in M^{1}\left(\mathbf{R}^{n}\right)$. We may therefore apply the Fubini Theorem to obtain

$$
J^{1}(\mu, \nu)=\frac{1}{2} \int\left[\iint \chi(x, y, h) d \mu(x) d \bar{\nu}(y)\right] d \eta(h) .
$$

But

$$
\iint \chi(x, y, h) d \mu(x) d \bar{\nu}(y)=\mu\left(H^{+}\right) \overline{\nu\left(H^{-}\right)}+\mu\left(H^{-}\right) \overline{\nu\left(\overline{\left.H^{+}\right)}\right.} .
$$

This proves (a). For (b), set $\nu=\mu$ and apply (a).

(4.4.5) Theorem. Suppose $\mu$ and $\nu$ are in $M^{1}\left(\mathbf{R}^{n}\right)$ and $V^{0}\left(\mathbf{R}^{n}\right)$. Then

(a) $J^{1}(\mu, \nu)=-\int \mu\left(H^{+}\right) \overline{\nu\left(H^{+}\right)} d \eta(h)$, and

(b) $I^{1}(\nu)=-\int\left|\nu\left(H^{+}\right)\right|^{2} d \eta(h)$.

Proof. Since $\mu\left(\mathbf{R}^{n}\right)=\nu\left(\mathbf{R}^{n}\right)=0$, and in view of Lemma (4.4.3), we have $\mu\left(H^{-}\right)=-\mu\left(H^{+}\right)$and $\nu\left(H^{-}\right)=-\nu\left(H^{+}\right)$for $\eta$-almost every $h$. The result follows from the previous theorem.

Concerning Theorem (4.4.5b), we remark that since $\nu\left(\mathbf{R}^{n}\right)=0$, the quantity $\left|\nu\left(H^{+}\right)\right|^{2}$ is independent of the orientation of $h$ for $\eta$-almost every $h$.

4.5. Applications to separation discrepancy. For a convex set $K$ in $\mathbf{R}^{n}$, define $T_{K}$ to be the collection of hyperplanes $h$ in $\mathbf{R}^{n}$ that have nonempty intersection with $K$. If $K$ is a ball of radius $R$, then from the definition of $\eta$ in the paragraph preceding Lemma (4.4.1), it follows that

$$
\eta\left(T_{K}\right)=c_{n} R,
$$

where $c_{n}$ is a positive dimensional constant. (In fact, $c_{n}$ is equal to the $\eta$ measure of the collection $T_{K_{0}}$, where $K_{0}$ is the ball of unit radius in $\mathbf{R}^{n}$.)

Now if $\nu$ is a signed measure of total mass zero having support within a ball of radius $R$, then we have the inequality

$$
-I^{1}(\nu) \leq c_{n} R\left[D_{S}(\nu)\right]^{2}
$$

due to Alexander [A2 and A3]. Here, $c_{n}$ is a positive dimensional constant. Theorem (4.4.5b) shows that this inequality holds also when $\nu$ is complexvalued. Indeed, we have

$$
\left|\nu\left(H^{+}\right)\right| \leq D_{S}(\nu)
$$

for every open half-plane $H^{+}$determined by a hyperplane $h$, and so if $K$ is the ball of radius $R$ that contains the support of $\nu$, then

$$
\int_{T_{K}}\left|\nu\left(H^{+}\right)\right|^{2} d \eta(h) \leq \int_{T_{K}}\left[D_{S}(\nu)\right]^{2} d \eta(h) .
$$

Now by equation (4.5.1),

$$
\int_{T_{K}}\left[D_{S}(\nu)\right]^{2} d \eta(h)=\eta\left(T_{K}\right)\left[D_{S}(\nu)\right]^{2}=c_{n} R\left[D_{S}(\nu)\right]^{2} .
$$

On the other hand, by Theorem (4.4.5b),

$$
\int_{T_{K}}\left|\nu\left(H^{+}\right)\right|^{2} d \eta(h)=-I^{1}(\nu) .
$$


(4.5.3) Theorem. Suppose the function $f$ on $\mathbf{R}^{n}$ is defined on $N^{n}$ open cubes $S_{i}$, each of side length $s$, arranged in an $N \times \cdots \times N$ lattice. Let $f$ have value $z_{i} \in \mathbf{C}$ on $S_{i}$, and suppose $\sum z_{i}=0$. If $d \nu=f d x$, then

$$
D_{S}(\nu) \geq c_{n} s^{n}\left(\frac{1}{N} \sum\left|z_{i}\right|^{2}\right)^{1 / 2},
$$

where $c_{n}$ is a positive dimensional constant independent of the location of the lattice and of the number, size, and weights of the cubes.

Proof. The support of $f$ is contained within a closed cube of side length $s N$, and this cube is contained in a ball $K$ of radius $\frac{1}{2} n^{1 / 2} s N$. Since by Theorem (4.2.1) we have

$$
-I^{1}(\nu) \geq c_{n}^{\prime} s^{2 n+1}\left(\sum\left|z_{i}\right|^{2}\right)
$$

we may apply (4.5.2) to obtain

$$
s N\left[D_{S}(\nu)\right]^{2} \geq c_{n}^{\prime \prime} s^{2 n+1}\left(\sum\left|z_{i}\right|^{2}\right)
$$

Thus,

$$
\left[D_{S}(\nu)\right]^{2} \geq c_{n}^{\prime \prime} s^{2 n}\left(\frac{1}{N} \sum\left|z_{i}\right|^{2}\right)
$$

(4.5.4) Theorem. Suppose that the real-valued function $f$ on $\mathbf{R}^{n}$ is continuously differentiable with support in a ball of radius $R$ and that $\int f=0$ but $f \neq 0$. If $d \nu=f d x$, then

$$
D_{S}(\nu) \geq c_{n} R^{-1 / 2}\||\operatorname{grad} f|\|_{\infty}^{-(2 n+1) / 2}\|f\|_{\infty}^{(2 n+3) / 2},
$$

where $c_{n}$ is a positive dimensional constant independent of $f$.

Proof. By Theorem (4.3.5),

$$
-I^{1}(\nu) \geq c_{n}^{\prime}\||\operatorname{grad} f|\|_{\infty}^{-(2 n+1)}\|f\|_{\infty}^{2 n+3} .
$$

Therefore by (4.5.2),

$$
\left[D_{S}(\nu)\right]^{2} \geq c_{n}^{\prime \prime} R^{-1}\||\operatorname{grad} f|\|_{\infty}^{-(2 n+1)}\|f\|_{\infty}^{2 n+3}
$$

Observe that Theorems (4.5.3) and (4.5.4) reduce to Theorems (1.1) and (1.2) when $n=2$.

4.6. Applications to the Radon transform. The Radon transform of a function $f \in L^{1}\left(\mathbf{R}^{n}\right)$ is the function $\mathscr{R} f$ on $S^{n-1} \times \mathbf{R}$ defined by

$$
\mathscr{R} f(\sigma, t)=\int_{\langle x, \sigma\rangle=t} f(x) d x,
$$

where the integration here is with respect to $(n-1)$-dimensional Lebesgue measure on the hyperplane $\{x:\langle x, \sigma\rangle=t\}$.

The following theorem shows the relationship between the Radon transform and the functional $-I^{1}$. 
(4.6.1) Theorem. Suppose $f \in L^{1}\left(\mathbf{R}^{n}\right)$ satisfies $\int f(x) d x=0$ and $\int|x f(x)| d x$ $<\infty$. If $d \nu=f d x$, then

$$
-I^{1}(\nu)=c_{n} \int_{S^{n-1}} \int_{-\infty}^{\infty}\left|\int_{-\infty}^{t} \mathscr{R} f(\sigma, r) d r\right|^{2} d t d \sigma,
$$

where $c_{n}$ is a positive dimensional constant.

Proof. The hypotheses give us that $\nu$ is in $M^{1}\left(\mathbf{R}^{n}\right)$ and $V^{0}\left(\mathbf{R}^{n}\right)$. Therefore by Theorem $(4.4 .5 \mathrm{~b})$, we have

$$
-I^{1}(\nu)=\int\left|\nu\left(H^{+}\right)\right|^{2} d \eta(h) .
$$

Now if $H^{+}=H^{+}(\sigma, t)=\{x:\langle x, \sigma\rangle<t\}$ is the open half-space determined by the oriented hyperplane $h=\{x:\langle x, \sigma\rangle=t\}$, then

$$
\nu\left[H^{+}(\sigma, t)\right]=\int_{-\infty}^{t} \int_{\langle x, \sigma\rangle=r} f(x) d x d r=\int_{-\infty}^{t} \mathscr{R} f(\sigma, r) d r .
$$

But since $d \eta$ is a constant multiple of $d \sigma d t$, we have

$$
-I^{1}(\nu)=c_{n} \int_{S^{n-1}} \int_{-\infty}^{\infty}\left|\nu\left[H^{+}(\sigma, t)\right]\right|^{2} d t d \sigma .
$$

A mixed norm of a function $F$ on $S^{n-1} \times \mathbf{R}$ can be defined for $1<q, p<\infty$ by

$$
\|F\|_{q, p}=\left(\int_{S^{n-1}}\left[\int_{\mathbf{R}}|F(\sigma, t)|^{p} d t\right]^{q / p} d \sigma\right)^{1 / q} .
$$

For $q=p$, this norm is the usual $L^{p}\left(S^{n-1} \times \mathbf{R}\right)$ norm. For $p=\infty$, define

$$
\|F\|_{q, \infty}=\left(\int_{S^{n-1}}[\underset{t \in \mathbf{R}}{\operatorname{ess} \sup }|F(\sigma, t)| d t]^{q} d \sigma\right)^{1 / q} .
$$

(4.6.2) Theorem. Suppose that the function $f \in L^{1}\left(\mathbf{R}^{n}\right)$ has support within the ball of radius $R$ centered at the origin and satisfies $\int f=0$. Let $d \nu=f d x$. If $1 \leq p \leq \infty$, then

$$
-I^{1}(\nu) \leq c_{n}(2 R)^{3-2 / p}\|\mathscr{R} f\|_{2, p}^{2},
$$

where $c_{n}$ is a positive dimensional constant.

Remark. Since $2 \leq 2^{3-2 / p} \leq 8$ for $1 \leq p \leq \infty$, the factor $2^{3-2 / p}$ can be absorbed into the constant $c_{n}$, so that (4.6.3) can be written alternatively as

$$
-I^{1}(\nu) \leq c_{n} R^{3-2 / p}\|\mathscr{R} f\|_{2, p}^{2} .
$$

Proof. First assume $1<p<\infty$. By Theorem (4.6.1),

$$
-I^{1}(\nu)=c_{n} \int_{S^{n-1}} \int_{-R}^{R}\left|\int_{-R}^{t} \mathscr{R} f(\sigma, r) d r\right|^{2} d t d \sigma .
$$

Now by Hölder's Inequality on the interval $[-R, t]$, we have for $p^{\prime}=p /(p-1)$ that

$$
\int_{-R}^{t}|\mathscr{R} f(\sigma, r)| d r \leq\left(\int_{-R}^{t}|\mathscr{R} f(\sigma, r)|^{p} d r\right)^{1 / p}\left(\int_{-R}^{t} 1^{p^{\prime}} d r\right)^{1 / p^{\prime}} .
$$


It follows that

$$
\begin{aligned}
-I^{1}(\nu) & \leq c_{n} \int_{S^{n-1}} \int_{-R}^{R}\left(\int_{-R}^{R}|\mathscr{R} f(\sigma, r)|^{p} d r\right)^{2 / p}(2 R)^{2 / p^{\prime}} d t d \sigma \\
& \leq c_{n}(2 R)^{2 / p^{\prime}+1} \int_{S^{n-1}}\left(\int_{-R}^{R}|\mathscr{R} f(\sigma, r)|^{p} d r\right)^{2 / p} d \sigma \\
& \leq c_{n}(2 R)^{3-2 / p}\|\mathscr{R} f\|_{2, p}^{2} .
\end{aligned}
$$

For the $p=1$ and $p=\infty$ cases, modify the argument appropriately.

We now show how precise the result of Theorem (4.6.2) is for the case $n=2$. We shall make use of the following lemma.

(4.6.4) Lemma. Given $R>0$, there exists a positive, radial function $g_{R} \in$ $L^{1}\left(\mathbf{R}^{2}\right)$ that has support within the disk of radius $R$ centered at the origin and whose Radon transform has constant value one on lines intersecting this disk.

Proof. Let

$$
g_{R}(x)= \begin{cases}\frac{1}{\pi \sqrt{R^{2}-|x|^{2}}}, & |x|<R, \\ 0, & |x| \geq R .\end{cases}
$$

Then for all $t<R$ and all $\sigma \in S^{1}$,

$$
\mathscr{R} g_{R}(\sigma, t)=\frac{2}{\pi} \int_{0}^{\sqrt{R^{2}-t^{2}}} \frac{d s}{\sqrt{R^{2}-t^{2}-s^{2}}}=1 \text {. }
$$

The positive function $g_{R}$ is indeed in $L^{1}\left(\mathbf{R}^{2}\right)$, since (taking any $\sigma \in S^{1}$ ) we have

$$
\int g_{R}(x) d x=\int_{-R}^{R} \mathscr{R} g_{R}(\sigma, t) d t=2 R .
$$

(4.6.5) Theorem. Given $R>0$, there exists $f \in L^{1}\left(\mathbf{R}^{2}\right)$ having support within the disk of radius $R$ centered at the origin with $\int f=0$, such that if $d \nu=f d x$ and $1 \leq p \leq \infty$, then

$$
c(2 R)^{3-2 / p}\|\mathscr{R} f\|_{2, p}^{2} \leq-I^{1}(\nu)
$$

where $c$ is a positive constant independent of $p$ and $R$.

Remark. As with Theorem (4.6.2), we have $2 \leq 2^{3-2 / p} \leq 8$ for $1 \leq p \leq \infty$, so that the factor $2^{3-2 / p}$ can be absorbed into the constant $c$. Thus, the conclusion of this theorem can be written alternatively as

$$
c R^{3-2 / p}\|\mathscr{R} f\|_{2, p}^{2} \leq-I^{1}(\nu) .
$$

Proof. Let $f=R^{-1} g_{R}-2 R^{-1} g_{R / 2}$, where $g_{R}$ and $g_{R / 2}$ are as in Lemma (4.6.4). For any $\sigma \in S^{1}$, we have

$$
\mathscr{R} f(\sigma, t)= \begin{cases}-R^{-1}, & |t|<\frac{1}{2} R, \\ R^{-1}, & \frac{1}{2} R \leq|t|<R, \\ 0, & |t| \geq R .\end{cases}
$$


Thus, for any $\sigma \in S^{1}$,

$$
\int_{-R}^{t} \mathscr{R} f(\sigma, r) d r= \begin{cases}0, & t<-R, \\ R^{-1} t+1, & -R \leq t<-\frac{1}{2} R, \\ -R^{-1} t, & -\frac{1}{2} R \leq t<\frac{1}{2} R, \\ R^{-1} t-1, & \frac{1}{2} R \leq t<R, \\ 0, & t \geq R .\end{cases}
$$

Writing $F(t)=\int_{-R}^{t} \mathscr{R} f(\sigma, r) d r$, we have $\int_{-R}^{R}[F(t)]^{2} d t=\frac{1}{6} R$. Therefore by Theorem (4.6.1),

$$
-I^{1}(\nu)=c_{2} \int_{S^{1}} \int_{-R}^{R}\left(\int_{-R}^{t} \mathscr{R} f(\sigma, r) d r\right)^{2} d t d \sigma=\frac{1}{6} c_{2}(2 \pi) R=c^{\prime} R .
$$

On the other hand, $|\mathscr{R} f(\sigma, t)| \equiv R^{-1}$ for $t<R$, so that for $1 \leq p<\infty$ we have

$$
\left(\int_{-R}^{R}|\mathscr{R} f(\sigma, t)|^{p} d t\right)^{1 / p}=R^{-1}(2 R)^{1 / p},
$$

while the $p=\infty$ case is

$$
\underset{-R \leq t \leq R}{\operatorname{ess} \sup }|\mathscr{R} f(\sigma, t)|=R^{-1} .
$$

In either case,

$$
\|\mathscr{R} f\|_{2, p}^{2}=\int_{\sigma \in S^{1}} R^{-2}(2 R)^{2 / p}=2 \pi R^{-2}(2 R)^{2 / p},
$$

and so $(2 R)^{3-2 / p}\|\mathscr{R} f\|_{2, p}^{2}=16 \pi R$.

We now apply Theorem (4.6.2) to the specific examples of Theorems (4.2.1) and (4.3.5).

(4.6.6) Theorem. Suppose the function $f$ on $\mathbf{R}^{n}$ is defined on $N^{n}$ open cubes $S_{i}$, each of side length $s$, arranged in an $N \times \cdots \times N$ lattice. Let $f$ have value $z_{i} \in \mathbf{C}$ on $S_{i}$, and suppose $\sum z_{i}=0$. If $1 \leq p \leq \infty$, then

$$
\|\mathscr{R} f\|_{2, p} \geq c_{n}(\sqrt{n} N)^{-3 / 2+1 / p} s^{n-1+1 / p}\left(\sum\left|z_{i}\right|^{2}\right)^{1 / 2}
$$

where $c_{n}$ is a positive dimensional constant independent of the location of the lattice and of the number, size, and weights of the cubes.

Proof. Let $d \nu=f d x$. The support of $f$ is contained within a closed cube of side length $s N$, and this cube is contained in a ball of radius $R=\frac{1}{2} n^{1 / 2} s N$. By Theorem (4.2.1),

$$
-I^{1}(\nu) \geq c_{n}^{\prime} s^{2 n+1}\left(\sum\left|z_{i}\right|^{2}\right)
$$

so we may apply Theorem (4.6.2) to obtain

$$
(2 R)^{3-2 / p}\|\mathscr{R} f\|_{2, p}^{2} \geq c_{n}^{\prime \prime} s^{2 n+1}\left(\sum\left|z_{i}\right|^{2}\right) \text {. }
$$

Therefore

$$
\|\mathscr{R} f\|_{2, p}^{2} \geq c_{n}^{\prime \prime}(2 R)^{-3+2 / p} s^{2 n+1}\left(\sum\left|z_{i}\right|^{2}\right) .
$$

Taking square roots proves the theorem. 
(4.6.7) Theorem. Suppose that the real-valued function $f$ on $\mathbf{R}^{n}$ is continuously differentiable with support in a ball of radius $R$ and that $\int f=0$ but $f \not \equiv 0$. Then for $1 \leq p \leq \infty$,

$$
\|\mathscr{R} f\|_{2, p} \geq c_{n} R^{-3 / 2+1 / p}\|\mid \operatorname{grad} f\|_{\infty}^{-(2 n+1) / 2}\|f\|_{\infty}^{(2 n+3) / 2}
$$

where $c_{n}$ is a positive dimensional constant independent of $f$.

Proof. Let $d \nu=f d x$. By Theorem (4.3.5),

$$
-I^{1}(\nu) \geq c_{n}^{\prime}\||\operatorname{grad} f|\|_{\infty}^{-(2 n+1)}\|f\|_{\infty}^{2 n+3},
$$

so by Theorem (4.7.4) (and the remark following it) we have

$$
R^{3-2 / p}\|\mathscr{R} f\|_{2, p}^{2} \geq c_{n}^{\prime \prime}\||\operatorname{grad} f|\|_{\infty}^{-(2 n+1)}\|f\|_{\infty}^{2 n+3} \text {. }
$$

Thus,

$$
\|\mathscr{R} f\|_{2, p}^{2} \geq c_{n}^{\prime \prime} R^{-3+2 / p}\||\operatorname{grad} f|\|_{\infty}^{-(2 n+1)}\|f\|_{\infty}^{2 n+3} .
$$

Observe that Theorems (4.6.6) and (4.6.7) reduce to the inequalities (1.12) and (1.13) when $p=2$ and $n=2$.

\section{REFERENCES}

[A1] R. Alexander, Generalized sums of distances, Pacific J. Math. 56 (1975), 297-304.

[A2] Geometric methods in the study of irregularities of distribution, Combinatorica 10 (1990), 115-136.

[A3] _ Principles of a new method in the study of irregularities of distribution, Invent. Math. 103 (1991), 279-296.

[AS] R. Alexander and K. B. Stolarsky, Extremal problems of distance geometry related to energy integrals, Trans. Amer. Math. Soc. 193 (1974), 1-31.

[Be1] J. Beck, On a problem of K. F. Roth concerning irregularities of point distribution, Invent. Math. 74 (1983), 477-487.

[Be2] __ Sums of distances between points on a sphere-an application of the theory of irregularities of distribution to discrete geometry, Mathematika 31 (1984), 33-41.

[BC] J. Beck and W. W. L. Chen, Irregularities of distribution, Cambridge Tracts in Math., vol. 89, Cambridge Univ. Press, London and New York, 1987.

[Bj] G. Björck, Distributions of positive mass, which maximize a certain generalized energy integral, Ark. Mat. 3 (1956), 255-269.

[Bo] S. Bochner, Harmonic analysis and the theory of probability, Univ. of California Press, Berkeley and Los Angeles, 1955.

[D] S. R. Deans, The Radon transform and some of its applications, Wiley-Interscience, New York, 1983.

[DS] N. Dunford and J. T. Schwartz, Linear operators. I. General theory, Pure and Appl. Math., vol. 7, Interscience, New York, 1958.

[E] P. Erdős, Problems and results on diophantine approximations, Compositio Math. 16 (1964), 52-65.

[Fo] G. B. Folland, Real analysis. Modern techniques and their applications, Pure and Appl. Math., Wiley-Interscience, New York, 1984.

[Fr] O. Frostman, Potentiel d'équilibre et capacité des ensembles avec quelques applications à la théorie des fonctions, Medd. Lunds Univ. Mat. Sem. 3 (1935), 1-118.

[Hl] S. Helgason, Groups and geometric analysis. Integral geometry, invariant differential operators, and spherical functions, Pure and Appl. Math., vol. 113, Academic Press, San Diego, CA, 1984. 
[Hr] A. Hertle, Continuity of the Radon transform and its inverse on Euclidean space, Math. Z. 184 (1983), 165-192.

[Kn] L. Kuipers and H. Niederreiter, Uniform distribution of sequences, Pure and Appl. Math., Wiley-Interscience, New York, 1974.

[OS] D. M. Oberlin and E. M. Stein, Mapping properties of the Radon transform, Indiana Univ. Math. J. 31 (1982), 641-650.

[PS] G. Pólya and G. Szegö, Über den transfiniten Durchmesser (Kapazitätskonstante) von ebenen und räumlichen Punktmengen, J. Reine Angew. Math. 165 (1931), 4-49.

[Ra] J. Radon, Über die Bestimmung von Funktionen durch ihre Integralwerte längs gewisser Mannigfaltigkeiten, Ber. Verh. Sächs. Akad. Wiss. Leipzig. Math.-Phys. Kl. 69 (1917), 262-277.

[Rg] A. D. Rogers, Theory and applications of a functional from metric geometry, Ph.D. thesis, Univ. of Illinois at Urbana-Champaign, 1990.

[Rt] K. F. Roth, On irregularities of distribution, Mathematika 1 (1954), 73-79.

[Ru] W. Rudin, Real and complex analysis, 3rd ed., McGraw-Hill, New York, 1987.

[Sa] L. A. Santaló, Integral geometry and geometric probability, Encyclopedia of Mathematics and Its Applications, vol. 1, Addison-Wesley, Reading, MA, 1976.

[Sm1] W. M. Schmidt, Irregularities of distribution. IV, Invent. Math. 7 (1969), 55-82.

[Sm2] _ Lectures on irregularities of distribution, Tata Inst. Fund. Res. Lectures on Math. and Phys., vol. 56, Tata Inst. Fund. Res., Bombay, 1977.

[Sol] I. J. Schoenberg, On ci:tain metric spaces arising from Euclidean spaces by a change of metric and their imbedding in Hilbert space, Ann. of Math. (2) 38 (1937), 787-793.

[So2] _ Metric spaces and positive definite functions, Trans. Amer. Math. Soc. 44 (1938), 522-536.

[St] K. B. Stolarsky, Sums of distances between points on a sphere. II, Proc. Amer. Math. Soc. 41 (1973), 575-582.

[Wa] G. Wagner, On means of distances on the surface of a sphere (lower bounds), Pacific J. Math. 144 (1990), 389-398.

[We] H. Weyl, Über die Gleichverteilung von Zahlen mod. Eins, Math. Ann. 77 (1916), 313-352.

Department of Mathematics, Elmhurst College, Elmhurst, Illinois 60126

E-mail address: allenr@elmhurst.edu 